\title{
Insight into the Characteristics of Research Published in Complementary, Alternative, and Integrative Medicine Journals: A Bibliometric Analysis
}

Jeremy Y. Ng ( NGJY2@MCMASTER.CA)

McMaster University

\section{Research Article}

Keywords: bibliometric analysis, complementary and alternative medicine, integrative health, integrative medicine, research trends, scientometrics

Posted Date: April 21st, 2021

DOI: https://doi.org/10.21203/rs.3.rs-440363/v1

License: @ (i) This work is licensed under a Creative Commons Attribution 4.0 International License. Read Full License

Version of Record: A version of this preprint was published on July 1st, 2021. See the published version at https://doi.org/10.1186/s12906-021-03354-7. 


\section{Abstract}

Background: Complementary, alternative and integrative medicine (CAIM) can be described as diverse medical and healthcare interventions, practices, products, or disciplines that are not considered as part of conventional medicine. Inherent in its definition, CAIMs are comprised of a wide variety of therapies with highly variable safety and effectiveness evidence profiles. Despite this, the use of many CAIMs is highly prevalent among patients globally. The present study consists of a bibliometric analysis of CAIM journals.

Methods: A single search of all International Standard Serial Number (ISSNs) of all journals categorized as "complementary and alternative medicine" (code 2707) based on the All Science Journal Classification (ASJC) was run on Scopus on April 17, 2021. All publication types were included; no further search limits were applied. The following bibliometric data were collected: number of publications (in total and per year), authors and journals; open access status; journals publishing the highest volume of literature and their impact factors; language, countries, institutional affiliations, and funding sponsors of publications; most productive authors; and highest-cited publications. Trends associated with this subset of publications were identified and presented. Bibliometric networks were constructed and visualized using the software tool VoSviewer.

Results: A total of 172466 publications (42331 open access), were published by 219680 authors in 143 journals from 1938 to 2021 . Since the 1940 s, an upward trend with respect to the volume of publications can be observed, with a steep increase observed between the mid-2000s and mid-2010s. The journal that published the largest number of publications was the Journal of Natural Products $(n=15144)$. The most productive countries included China ( $n=45860)$, the United States $(n=29523)$, and Germany $(n=10120)$; a number of the most common intuitional affiliations and funding sponsors also originated from these three countries.

Conclusions: The number of publications collectively published in CAIM journals follows an upward trend. Given a high prevalence of CAIM use among patients, increased acceptance of CAIM among conventional healthcare providers, and growing interest in the research of CAIM, future work should continue to investigate and track changes in the publication characteristics of the emerging research on this topic.

\section{Background}

Complementary and alternative medicine is generally defined as a group of diverse medical and healthcare interventions, practices, products or disciplines that are not considered as part of conventional medicine [1]. Specifically, the National Center for Complementary and Integrative Health (NCCIH) defines "complementary" medicine as a non-mainstream practice used together with conventional medicine, whereas "alternative" medicine refers to a nonmainstream practice used in place of conventional medicine [2]. In contrast, "integrative health" is defined as the coordinated delivery of conventional and complementary approaches together [2]. These three words - complementary, alternative, and integrative - comprise the most common ways to refer to these types of therapies [3]. For the purpose of the present study, these therapies will be referred to collectively as "complementary, alternative and integrative medicine" or "CAIMs" hereafter. At present, a lack of consensus exists regarding how to categorize CAIMs; in fact, by definition every therapy that falls under the umbrella of "CAIM" exists as a result of being considered outside of the purview of conventional Western medical practices [3]. Therefore, one CAIM therapy can be highly unrelated to another due to the fact that each originates from a different region in the world, culture, system of traditional medicine, and school of thought [4,5]. Despite these challenges, attempts have been made to categorize CAIMs. For example, the NCCIH divides CAIM therapies into two main types: 1) natural products and 2) mind and body practices. Within the former category, they include therapies such as herbs, vitamins and minerals, and probiotics, while in the latter, they include therapies such as yoga, chiropractic and osteopathic manipulation, and meditation, as well as acupuncture, relaxation techniques, tai chi, qi gong, and hypnotherapy, among others [2]. Despite these efforts, the NCCIH has stated, however, that some CAIMs may not fit neatly into either of these two groups, citing many systems of CAIM including practices of traditional healers, Ayurvedic medicine, traditional Chinese medicine, homeopathy, naturopathy, and functional medicine [2].

Regardless of how CAIMs are categorized, these therapies are perceived to be of value by their proponents for their emphasis on a holistic, patient-focused approach to healthcare, which include mental, emotional, functional, spiritual, economic, and social aspects [2,6]. CAIM is widely used around the world, with $88 \%$ of World Health Organization member states acknowledging their use of CAIM, which by definition means that these 170 countries have formally developed policies, laws, regulations, programs and offices for CAIM [4]. The prevalence of CAIM use is high in many Western countries; for example, it is estimated that around $80 \%$ of Canadians have used CAIM [7]. The prevalence of CAIM use is also documented to be high among certain patient populations; in cancer patients, as many as $90 \%$ report using some type of CAIM $[8,9,10]$. CAIM is used by these patients for a variety of reasons, including symptom relief, improved quality of life, supplementing conventional therapy, supporting one's philosophical orientations toward health, and achieving a sense of control over one's care $[11,12,13]$. Integrative medicine (the use of complementary and conventional therapies) is becoming increasingly popular among patients and practitioners [14,15], and sub-specializations of integrative care for specific diseases/conditions have also been established, such as integrative oncology $[16,17]$.

While some CAIMs, such as meditation and yoga $[18,19]$, have undergone more thorough testing and have been found to be generally safe and effective, others have not been adequately researched to determine their effectiveness, and some have been found to be potentially harmful or interact negatively with conventional medicines [20,21,22]. The belief among patients that "natural means safe and better" [23] is well-documented, however, evidence from the research literature suggests otherwise. Many herbal and dietary supplements can be harmful when taken in large quantities. Certain weight loss and bodybuilding supplements have been shown to cause hepatotoxicity or even hepatic failure at therapeutic doses [24]. Furthermore, systemized pharmacovigilance of CAIMs is poorly coordinated on a national and international level, and CAIM therapies are generally not held to the same standards of regulation as that of pharmaceutical medicines in terms of quality, effectiveness, and safety [25].

The increase in popularity and prevalence of CAIM use among patients, and growing acknowledgement among conventional healthcare providers that a need exists to approach CAIM therapies, their traditions, and their practitioners with respect, are among some of the reasons for an increase in CAIM research 
productivity which has resulted in a growth in the volume of the published literature over the past few decades $[26,27,28]$. The application of a research method known as a bibliometric analysis can facilitate a better understanding of a given field, such as that of CAIM. A bibliometric analysis involves the statistical assessment of scientific publications, to identify the characteristics and determine the impact of the literature published in a specific academic discipline $[29,30,31]$. This increased interest in CAIM research has led to the establishment and indexing of multiple CAIM journals, however, no study has comprehensively assessed the characteristics of the publications found within these source titles to date. Thus, the purpose of the present study is to provide current insight into the characteristics of publications published across CAIM journals through a bibliometric analysis.

\section{Methods \\ Publication Search and Characteristics}

The 2021 Scopus Source List [32] was downloaded, and all Scopus-indexed journals belonging to the "complementary and alternative medicine" category (code 2707) were identified based on the All Science Journal Classification (ASJC). A single search containing the International Standard Serial Numbers (ISSNs) of all of these journals was run on Scopus on April 17, 2021; the search strategy can be found in Table 1. Search results were exported on the same day to prevent discrepancies between daily database updates. Searches were only conducted on Scopus because it is the largest abstract and citation database of peer-reviewed literature [33]; in comparison, Web of Science contains considerably fewer CAIM-categorized journals, while OVID databases do not provide certain metrics such as publication citation counts [34]. All publication types were included, and no further search limits were applied. The following bibliometric data were collected: number of publications (in total and per year), authors and journals; open access status; journals publishing the highest volume of literature and their impact factors; language, countries, institutional affiliations, and funding sponsors of publications; most productive authors; and highest-cited publications. Trends associated with this subset of publications were identified and presented. Bibliometric networks were constructed and visualized using the software tool VOSviewer (version 1.6.16) [35,36].

\section{Results}

A total of 172466 publications (42331 open access), were published by 219680 authors in 143 journals from 1938 to 2021 . Since the 1940 s, an upward trend with respect to the volume of publications can be observed, with a steep increase observed between the mid-2000s and mid-2010s. This upward trend has continued with 2020 marking the year with the highest number of publications to date; Figure 1 depicts the number of publications published per year. The Journal of Natural Products ( $n=15144$ ) published the largest number of publications, followed by Zhongguo Zhongyao Zazhi ( $n=14577)$, and Planta Medica $(n=10793)$. All journals included within this bibliometric analysis were hand-searched on InCites Journal Citation Reports [37]. As of 2020, 83 journals were still active (57.6\%), of which 35 had a 2019 impact factor (range from 0.200 to 5.487). Table 2 provides complete details of the journals included in this bibliometric analysis, including the journal name, ISSN, whether the journal is active or inactive (as of 2020), coverage period, title history indication, publisher name, number of publications published, and the 2019 impact factor (if available).

The subject area containing the largest number of publications was medicine ( $n=172456)$, followed by pharmacology, toxicology and pharmaceutics $(n=86902)$, then biochemistry, genetics and molecular biology $(n=40262)$. Publications were primarily published in English ( $n=135718)$, followed by Chinese $(n=24614)$, then German $(n=8611)$. The most common document types were article $(n=139540)$ and review $(n=13418)$. The most productive countries included China $(n=45860)$, the United States $(n=29523)$, and Germany $(n=10120)$. The most common affiliations were the China Academy of Chinese Medical Sciences ( $n=3560)$, the Beijing University of Chinese Medicine ( $n=2896)$, and the Chinese Academy of Sciences ( $n=2896)$; the most common funding sponsors were the National Natural Science Foundation of China $(n=5711)$, the National Institutes of Health $(n=4055)$, and the US Department of Health and Human Services $(n=4032$ ). The general characteristics of eligible publications are summarized in Table 3 . In addition, the 100 most highly published authors are provided in Table 4, and the 100 highest-cited publications are provided in Table 5.

Figures 2-5 represent bibliometric networks constructed and visualized using the software tool VoSviewer, and include all 172466 captured by the present study's search. This added layer of analysis of the most influential subset of publications captured provides a greater understanding of the relationship that exists between certain items (i.e. countries, keywords, authors, journals, etc). In each bibliometric network (figure), each item is represented in a network visualisation by a label and a circle; the weight of an item determines the size of the label and the circle of an item. Figure 2 depicts a co-authorship analysis of the 50 most productive countries. In a co-authorship analysis, the relatedness of items is determined based on the number of co-authored publications. From this figure, it can be seen that while China is the most productive country, Chinese authors tend to collaborate less with researchers in other countries as shown by the distance between lines. In contrast, American authors tend to collaborate with many countries internationally, while German authors tend to collaborate more with researchers in other European countries. Figure 3 depicts a co-occurrence analysis of the 500 most frequent author keywords used across all publications. In a co-occurrence analysis, the relatedness of items is determined based on the number of publications in which they occur together. From this figure, a number of clusters can be observed representing different CAIM topics. The yellow, red and dark blue clusters represent a large network of keywords related to laboratory-based studies, while the green cluster represents keywords related to clinical research and review-type studies. The smaller light blue cluster also highlights research conducted on traditional and indigenous medicines. This figure also provides insights into some of the most highly studied diseases/conditions published in CAIM journals, which include breast and lung cancer, diabetes, anxiety, and low back pain. Figures $\mathbf{4}$ and $\mathbf{5}$ depict a citation analysis of the 500 highest-cited authors, and the 100 sources publishing the largest number of CAIM publications, respectively. In a citation analysis, the relatedness of items is determined based on the number of times they cite each other.

\section{Discussion}

The objective of the present bibliometric analysis is to capture the characteristics of the research literature published in CAIM journals. The search conducted on Scopus yielded over 170000 publications, representing the largest bibliometric analysis of CAIM literature to date to the author's knowledge. Since the 
1940s, an upward trend with respect to the volume of publications can be observed, with a steep increase observed between the mid-2000s and mid-2010s. This upward trend has continued with 2020 marking the most productive year globally to date. This growth in the volume of research published over the most recent decades can largely be explained by an increase in funding support by government and nongovernment sectors for CAIM research [38,39,40,41]. In the present study, it was found that China was the most productive country with respect to CAIM research at 45860 publications, followed by the United States at 29523 and Germany at 10120. A vast amount of research continues to be conducted on traditional Chinese medicine in China [42,43,44], while the United States and Germany have both historically been the leading countries with respect to the research of various CAIM therapies $[45,46,47,48]$. While the vast majority of publications were written in English, which is largely regarded as the international language of academic publication, it is also unsurprising that the second most common language was Chinese, and the third was German, as this corresponds with the national languages of the most productive countries. Of the top 20 institutional affiliations responsible for publishing this CAIM research, 17 originated from China, with the remaining two from South Korea and one from Taiwan; a number of affiliations based in the United States and Germany existed as well, but below the top 20. Additionally, with respect to the top 20 funding sponsors, the countries with the largest number were China and the United States, with six organizations each.

\section{Comparative Literature}

The findings from a small number of published bibliometric analyses specific to the CAIM research literature can be compared to that of the present study. One of the first bibliometric analyses of the CAIM research literature was published by Barnes et al. in 1999. Using a number of CAIM-related keywords, they conducted searches on Medline and analysed the literature published from 1966 to 1996. At the time, they reported that the volume of CAIM publications per year rose between 1972 and 1986, and then remained stable and approximated 1500 per year up until 1996. Although in the present study a growth in the volume of literature is still observed from 1986 to 1996, the mean number of publications per year over this decade was approximately 1400 , which aligns closely with the findings of the authors [45]. Fu et al. (2011) analysed 17002 publications found in 19 complementary and alternative medicine journals over approximately three decades. They found that the most productive countries included the United States, China, India, England and Germany, all of which fell within the top seven most productive countries in the present study. A number of institutions were also identified by both Fu et al. (2011) as well as the present study to be among the most productive internationally, including China Medical University and Kyung Hee University [46]. Danell et al. analysed four decades' worth of complementary and alternative medicine publication activity from 1966-2007 [47], then later repeated their study again to include five decades from 1966-2016 [48]. In their more recent study, they analyzed 105216 publications, which prior to the present study was the largest bibliometric analysis on this topic. Unlike the present study which sought to characterize publications in CAIM journals, Danell et al.'s (2020) inclusion criteria included publications that had "Complementary Therapies" as their Medical Subject Heading major topic, in the Medline database. Lastly, Youn et al. (2021) conducted a bibliometric analysis of the integrative medicine research literature based on a search query using two keywords joined by the Boolean operator "OR": "complementary and integrative medicine" OR "integrative medicine", retrieving and analysing a total of 4660 publications. Although their study's focus was more so on integrative medicine, a number of their findings are shared with the present study; for example, they also identified United States, China, and Germany to be the most productive countries (albeit in this order), and they also found that cancer was one of the most commonly studied diseases/conditions [49].

In line with the findings made by Barnes et al. (1999) [45], Danell et al. (2009) [47], Fu et al. (2011) [46], Danell et al. (2020) [48], and Youn et al. (2021) [49], the present study also found an upward trend with respect to the volume of CAIM research being published each year over the past decades. With respect to the number of publications captured, although Danell et al.'s (2020) study was published in 2020, their coverage of the CAIM literature only extended up until 2016 [48]. In the present study, over 37000 publications were found to be published between 2017 and April 2021, comprising over $20 \%$ of the entire body of literature analysed.

\section{Future Directions}

Beyond the aforementioned comparative literature, it is worth noting that it has been far more common for bibliometric analyses to be conducted on a specific CAIM-related topic. These have included acupuncture [50,51,52], aromatherapy [53], apitherapy [54], complementary and integrative oncology [55], ethnopharmacology [56], homeopathy [57], medicinal plants [58], qi gong [59], and yoga [60,61], as just some examples among others. Others have conducted bibliometric analyses specific to methodologies, such as clinical trials $[60,62,63]$ or guidelines [51] in CAIM. Bibliometric analyses of the CAIM literature with specific sub-topics are more straightforward to conduct, as the keywords and searches applied are likewise also easier to standardize. One of the main challenges in conducting comprehensive bibliometric analyses of the CAIM literature in its entirety is the fact that it is very difficult to operationalize a dynamic and unrelated group of therapies that have been defined on the basis that they lie outside of the purview of conventional Western medical care [64,65]. As a result, all of the bibliometric analyses of the CAIM literature to date have been based on searches of CAIM-specific journals or CAIM-specific indexed headings, both of which unquestionably provide an incomplete picture of all the CAIM literature. Thus, future directions of value include 1) the creation of an operational definition of CAIM informed by a systematic search strategy, and 2) the development of standardized search strategies for major academic databases based on this operational definition.

\section{Strengths and Limitations}

This present bibliometric study captured and analysed the characteristics of over 170000 publications, making it the largest conducted to date with respect to the CAIM literature, and the most comprehensive with regards to CAIM journal inclusion. Searches were conducted on Scopus as this academic database has a larger coverage in comparison to other databases such as Web of Science. Despite this, it must be acknowledged that all academic databases contain gaps in their indexing, and this was realized at the point of analysis in the present study. Furthermore, it is always possible that some literature may not have been captured by not searching other databases, however, this would have introduced considerable complexities with respect to the ability to analyse search results efficiently (i.e. deduplication of such a large volume of publications, bibliometric network visualizations). The use of the software tool vosviewer to create and 
visualize bibliometric networks serves as an additional strength to the present study, providing a deeper layer of analysis with respect to the strength and nature of relationships between different items (countries, keywords, authors, journals). One final limitation includes the fact that independent search results were not screened as this would have been impractical, and possibly unfeasible without the application of an operational definition of CAIM. Without doing this, however, it is possible that this analysis also included non-CAIM literature published in journals categorized as "complementary and alternative medicine" by Scopus.

\section{Conclusions}

The present study provides current insight into the characteristics of publications published across CAIM journals, and represents the largest bibliometric analysis conducted to date with respect to the CAIM literature. The most productive countries included China, the United States, and Germany; unsurprisingly, a large proportion of common institutional affiliations and funding sponsors associated with this subset of publications also originated from these countries. The volume of publications has increased steadily since the 1940s, and a steep increase was observed between the mid-2000s and mid-2010s, which is largely attributable to increased available funding for CAIM research globally. This upward trend has continued with 2020 marking the year with the most publications to date. Beyond identifying the large diversity of CAIMs studied, this study also highlights therapies which may be understudied and warrant further investigation. Given a high prevalence of CAIM use among patients, increased acceptance of CAIM among conventional healthcare providers, and growing interest in the research of CAIM, future work should continue to investigate and track changes in the publication characteristics of the emerging research on this topic. The creation of an operational definition of CAIM informed by a systematic search strategy, followed by the development of standardized search strategies for major academic databases based on this operational definition, may serve to achieve these goals more comprehensively.

\section{Declarations}

\section{Ethics Approval and Consent to Participate}

This study involved a bibliometric analysis of the literature only; it did not require ethics approval or consent to participate.

\section{Consent for Publication}

The author consents to this manuscript's publication.

\section{Availability of Data and Materials}

All data generated or analysed during this study are included in this published article.

\section{Competing Interests}

The author declares that they have no competing interests.

\section{Funding}

This study was not funded.

\section{Authors' Contributions}

JYN: conceptualized and designed the study, collected the data, interpreted and analysed the data, drafted the manuscript, and gave final approval of the version to be submitted.

\section{Acknowledgements}

None.

\section{Abbreviations}

ASJC: All Science Journal Classification

CAIM: complementary, alternative, and integrative medicine

$\mathrm{NCClH}$ : National Center for Complementary and Integrative Health

\section{References}


1. Ventola CL. Current issues regarding complementary and alternative medicine (CAM) in the United States: Part 1: The widespread use of CAM and the need for better-informed health care professionals to provide patient counseling. Pharmacy and Therapeutics. 2010 Aug;35(8):461.

https://www.ncbi.nlm.nih.gov/pmc/articles/PMC2935644/

2. National Center for Complementary and Integrative Health (NCCIH). [Internet]. Complementary, Alternative, or Integrative Health: What's In a Name?; 2018 [cited 2021 Apr 17]. Available from: https://www.nccih.nih.gov/health/complementary-alternative-or-integrative-health-whats-in-a-name

3. Ng JY, Boon HS, Thompson AK, Whitehead CR. Making sense of "alternative", "complementary", "unconventional" and "integrative" medicine: exploring the terms and meanings through a textual analysis. BMC Complementary and Alternative Medicine. 2016 Dec 1;16(1):134.

https://doi.org/10.1186/s12906-016-1111-3

4. World Health Organization. WHO global report on traditional and complementary medicine 2019. Institutional Repository for Information Sharing (IRIS); 2019 [cited 2021 Mar28]. 226 p. https://apps.who.int/iris/handle/10665/312342

5. Petri Jr RP, Delgado RE, McConnell K. Historical and cultural perspectives on integrative medicine. Medical Acupuncture. 2015 Oct 1;27(5):309-17. https://doi.org/10.1089/acu.2015.1120

6. World Health Organization. WHO Traditional Medicine Strategy 2014-2023. Geneva: World Health Organization; 2013.76 p. https://www.who.int/medicines/publications/traditional/trm_strategy14_23/en/

7. Esmail N. Complementary and Alternative Medicine: Use and Public Attitudes 1997, 2006, and 2016. Vancouver: Fraser Institute; 2017.87 p. https://www.fraserinstitute.org/sites/default/files/complementary-and-alternative-medicine-2017.pdf

8. Savas P, Robertson A, Beatty L, Hookings E, McGee M, Marker J, et al. Patient preferences on the integration of complementary therapy with conventional cancer care: Complementary therapy and cancer preferences. Asia-Pacific Journal of Clinical Oncology. 2016 Jun;12(2):e311-8.

https://doi.org/10.1111/ajco.12226

9. Begbie SD, Kerestes ZL, Bell DR. Patterns of alternative medicine use by cancer patients. Medical Journal of Australia. 1996 Nov;165(10):545-8. https://doi.org/10.5694/j.1326-5377.1996.tb138639.x

10. Adams M, Jewell AP. The use of complementary and alternative medicine by cancer patients. International Seminars in Surgical Oncology. 2007 Apr 30;4(1):10. https://doi.org/10.1186/1477-7800-4-10

11. Astin JA. Why patients use alternative medicine: results of a national study. JAMA: The Journal of the American Medical Association. 1998 May 20;279(19):1548. https://doi.org/10.1001/jama.279.19.1548

12. Kristoffersen AE, Stub T, Musial F, Fønneb $\varnothing$ V, Lillenes $O$, Norheim AJ. Prevalence and reasons for intentional use of complementary and alternative medicine as an adjunct to future visits to a medical doctor for chronic disease. BMC Complementary and Alternative Medicine. 2018 Dec;18(1):1-8. https://doi.org/10.1186/s12906-018-2179-8

13. McCaffrey AM, Pugh GF, O'Connor BB. Understanding patient preference for integrative medical care: Results from patient focus groups. Journal of General Internal Medicine. 2007 Oct 12;22(11):1500-5. https://doi.org/10.1007/s11606-007-0302-5

14. Snyderman R, Weil AT. Integrative medicine: Bringing medicine back to its roots. Archives of Internal Medicine. 2002 Feb 25;162(4):395-7. https://doi.org/10.1001/archinte.162.4.395

15. Maizes V, Rakel D, Niemiec C. Integrative medicine and patient-centered care. Explore. 2009 Sep 1;5(5):277-89. https://doi.org/10.1016/j.explore.2009.06.008

16. Society for Integrative Oncology. [Internet]. What is Integrative Oncology? [cited 2021 Apr 17]. Available from: https://integrativeonc.org/knowledgecenter/what-is-integrative-oncology

17. Yun H, Sun L, Mao JJ. Growth of integrative medicine at leading cancer centers between 2009 and 2016: A systematic analysis of NCl-designated comprehensive cancer center websites. JNCI Monographs. 2017 Nov 1;2017(52). https://doi.org/10.1093/jncimonographs/lgx004

18. National Center for Complementary and Integrative Health (NCCIH) [Internet]. Safe Use of Complementary Health Products and Practices; 2021 [cited 2021 Apr 17]. Available from: https://www.nccih.nih.gov/health/safety

19. Cramer H, Lauche R, Langhorst J, Dobos G. Yoga for depression: A systematic review and meta- Depression and Anxiety. 2013 Nov;30(11):1068-83. https://doi.org/10.1002/da.22166

20. Bellanger RA, Seeger CM, Smith HE. Safety of complementary and alternative medicine treatments and practices. In: Side Effects of Drugs Annual 2019 Jan 1 (Vol. 41, pp. 559-571). Elsevier. https://doi.org/10.1016/bs.seda.2019.06.004

21. Pratt M, Wieland S, Ahmadzai N, Butler C, Wolfe D, Pussagoda K, et al. A scoping review of network meta-analyses assessing the efficacy and safety of complementary and alternative medicine interventions. Systematic Reviews. 2020 Dec;9:1-25. https://doi.org/10.1186/s13643-020-01328-3

22. Rossi EG, Bellandi T, Picchi M, Baccetti S, Monechi MV, Vuono C, et al.. Patient safety in complementary medicine through the application of clinical risk management in the public health system. Medicines. 2017 Dec;4(4):93. https://doi.org/10.3390/medicines4040093

23. National Center for Complementary and Integrative Health (NCCIH). [Internet]. Natural Doesn't Necessarily Mean Safer, or Better; 2021. [cited 2021 Apr 17]. Available from: https://www.nccih.nih.gov/health/know-science/natural-doesnt-mean-better

24. Navarro VJ, Khan I, Björnsson E, Seeff LB, Serrano J, Hoofnagle JH. Liver injury from herbal and dietary supplements. Hepatology. 2017 Jan;65(1):363-73. https://doi.org/10.1002/hep.28813

25. Bellanger RA, Seeger CM, Smith HE. Safety of complementary and alternative medicine treatments and practices. In: Side Effects of Drugs Annual 2019 Jan 1 (Vol. 41, pp. 559-571). Elsevier. https://doi.org/10.1016/bs.seda.2019.06.004

26. Coulter I, Willis E. Explaining the growth of complementary and alternative medicine. Health Sociology Review. 2007 Oct 1;16(3-4):214-25.

https://doi.org/10.5172/hesr.2007.16.3-4.214

Page 6/43 
27. Jonas WB, Eisenberg D, Hufford D, Crawford C. The evolution of complementary and alternative medicine (CAM) in the USA over the last 20 years. Complementary Medicine Research. 2013;20(1):65-72. https://doi.org/10.1159/000348284

28. Walach H. Good Morning Future: Complementary Medicine's Next 25 Years. Complementary medicine research. 2018;25(1):4-6. https://doi.org/10.1159/000486887

29. Pritchard A. Statistical bibliography or bibliometrics. Journal of documentation. 1969 Dec 25;25(4):348-9.

30. Price DD. A general theory of bibliometric and other cumulative advantage processes. Journal of the American society for Information science. 1976 Sep;27(5):292-306. https://doi.org/10.1002/asi.4630270505

31. Hicks D, Wouters P, Waltman L, De Rijcke S, Rafols I. Bibliometrics: the Leiden Manifesto for research metrics. Nature News. 2015 Apr 23;520(7548):429. https://doi.org/10.1038/520429a

32. Scopus Preview [Internet]. Sources; 2021 [cited 2021 Apr 17]. Available from: https://www.scopus.com/sources.uri?zone=TopNavBar\&origin=searchbasic

33. Elsevier [Internet]. Scopus; 2021 [cited 2021 Apr 17]. Available from: https://www.elsevier.com/solutions/scopus

34. Gusenbauer M, Haddaway NR. Which academic search systems are suitable for systematic reviews or meta-analyses? Evaluating retrieval qualities of Google Scholar, PubMed, and 26 other resources. Research synthesis methods. 2020 Mar;11(2):181-217. https://doi.org/10.1002/jrsm.1378

35. Van Eck NJ, Waltman L. VOSviewer manual. Leiden: Univeristeit Leiden. November 2020. https://www.vosviewer.com/getting-started.

36. Van Eck NJ, Waltman L. Software survey: VOSviewer, a computer program for bibliometric mapping. Scientometrics. 2010 Aug 1;84(2):523-38. https://doi.org/10.1007/s11192-009-0146-3

37. Clarivate Analytics. [Internet]. InCites Journal Citation Reports; 2021 [cited 2021 Apr 17]. Available from: https://jcr.clarivate.com/

38. National Center for Complementary and Integrative Health (NCCIH) [Internet]. Complementary and Alternative Medicine Funding by NIH Institute/Center; 2019 [cited 2021 Apr 17]. Available from: https://www.nccih.nih.gov/about/budget/complementary-and-alternative-medicine-funding-by-nihinstitutecenter

39. Lewith G, Verhoef M, Koithan M, Zick SM. Developing CAM research capacity for complementary medicine. Evidence-based complementary and alternative medicine. 2006 Jun 1;3(2):283-9. https://doi.org/10.1093/ecam/nel007

40. Cyranoski D. Why Chinese medicine is heading for clinics around the world. Nature. 2018 Sep 1;561(7724):448-. https://doi.org/10.1038/d41586-01806782-7

41. World Health Organization [Internet]. Traditional, Complementary and Integrative Medicine; 2021 [cited 2021 Apr 17]. Available from: https://www.who.int/health-topics/traditional-complementary-and-integrative-medicine

42. Consentino R, Santos MJ, Matos LC, Machado JP. Bibliometric analysis of traditional Chinese medicine scientific production between 1982 and 2016 Indexed in PubMed. Medicines. 2018 Jun;5(2):41. https://doi.org/10.3390/medicines5020041

43. Huang Y, Zhou M, Deng Q, Zhang J, Zhou P, Shang X. Bibliometric analysis for the literature of traditional Chinese medicine in PubMed. Scientometrics. 2015 Oct;105(1):557-66. https://doi.org/10.1007/s11192-015-1686-3

44. Chen YB, Tong XF, Ren J, Yu CQ, Cui YL. Current research trends in traditional Chinese medicine formula: a bibliometric review from 2000 to 2016. Evidence-based complementary and alternative medicine. 2019 Mar 1;2019. https://doi.org/10.1155/2019/3961395

45. Barnes J, Abbot NC, Harkness EF, Ernst E. Articles on complementary medicine in the mainstream medical literature: an investigation of MEDLINE, 1966 through 1996. Archives of Internal Medicine. 1999 Aug 9;159(15):1721-5. https://doi.org/10.1001/archinte.159.15.1721

46. Fu JY, Zhang X, Zhao YH, Huang MH, Chen DZ. Bibliometric analysis of complementary and alternative medicine research over three decades. Scientometrics. 2011 Aug 1;88(2):617-26. https://doi.org/10.1007/s11192-011-0391-0

47. Danell JA, Danell R. Publication activity in complementary and alternative medicine. Scientometrics. 2009 Aug 1;80(2):539-51. https://doi.org/10.1007/s11192-008-2078-8

48. Danell JA, Danell R, Vuolanto P. Fifty Years of Complementary and Alternative Medicine (CAM): a Bibliometric Analysis of Publication Activity and General Content of the Publications. Journal of Scientometric Research. 2020;9(3):268-76. https://doi.org/10.5530/jscires.9.3.34

49. Youn BY, Song HJ, Yang K, Cheon C, Ko Y, Jang BH, Shin YC, Ko SG. Bibliometric Analysis of Integrative Medicine Studies from 2000 to 2019. American Journal of Chinese Medicine. 2021 Apr 7:1-3. https://doi.org/10.1142/S0192415X21500397

50. Ma Y, Dong M, Zhou K, Mita C, Liu J, Wayne PM. Publication trends in acupuncture research: a 20-year bibliometric analysis based on PubMed. PloS one. 2016 Dec 14;11(12):e0168123. https://doi.org/10.1371/journal.pone.0168123

51. Birch S, Lee MS, Alraek T, Kim TH. Overview of treatment guidelines and clinical practical guidelines that recommend the use of acupuncture: a bibliometric analysis. The Journal of Alternative and Complementary Medicine. 2018 Aug 1;24(8):752-69. https://doi.org/10.1089/acm.2018.0092

52. Lee IS, Lee H, Chen YH, Chae Y. Bibliometric analysis of research assessing the use of acupuncture for pain treatment over the past 20 years. Journal of Pain Research. 2020;13:367. https://doi.org/10.2147/JPR.S235047

53. Koo M. A bibliometric analysis of two decades of aromatherapy research. BMC research notes. 2017 Dec;10(1):1-9. https://doi.org/10.1186/s13104-0162371-1

54. Şenel E, Demir E. Bibliometric analysis of apitherapy in complementary medicine literature between 1980 and 2016. Complementary therapies in clinical practice. 2018 May 1;31:47-52. https://doi.org/10.1016/j.ctcp.2018.02.003

55. Moral-Munoz JA, Carballo-Costa L, Herrera-Viedma E, Cobo MJ. Production trends, collaboration, and main topics of the integrative and complementary oncology research area: a bibliometric analysis. Integrative cancer therapies. 2019 May;18:1534735419846401.

https://doi.org/10.1177/1534735419846401

Page $7 / 43$ 
56. Yeung AW, Heinrich M, Atanasov AG. Ethnopharmacology-a bibliometric analysis of a field of research meandering between medicine and food science?. Frontiers in Pharmacology. 2018 Mar 15;9:215. https://doi.org/10.3389/fphar.2018.00215

57. Chiu WT, Ho YS. Bibliometric analysis of homeopathy research during the period of 1991 to 2003. Scientometrics. 2005 Mar 1;63(1):3-23. https://doi.org/10.1007/s11192-005-0201-7

58. Salmerón-Manzano E, Garrido-Cardenas JA, Manzano-Agugliaro F. Worldwide research trends on medicinal plants. International journal of environmental research and public health. 2020 Jan;17(10):3376. https://doi.org/10.3390/ijerph17103376

59. Zhang YP, Hu RX, Han M, Lai BY, Liang SB, Chen BJ, Robinson N, Chen K, Liu JP. Evidence base of clinical studies on qi gong: a bibliometric analysis. Complementary Therapies in Medicine. 2020 May 1;50:102392. https://doi.org/10.1016/j.ctim.2020.102392

60. Cramer H, Lauche R, Dobos $\mathrm{G}$. Characteristics of randomized controlled trials of yoga: a bibliometric analysis. BMC complementary and alternative medicine. 2014 Dec;14(1):1-20. https://doi.org/10.1186/1472-6882-14-328

61. Jeter PE, Slutsky J, Singh N, Khalsa SB. Yoga as a therapeutic intervention: a bibliometric analysis of published research studies from 1967 to 2013 . The Journal of Alternative and Complementary Medicine. 2015 Oct 1;21(10):586-92. https://doi.org/10.1089/acm.2015.0057

62. Vickers AJ. Bibliometric analysis of randomized trials in complementary medicine. Complementary Therapies in Medicine. 1998 Dec 1;6(4):185-9. https://doi.org/10.1016/S0965-2299(98)80026-5

63. Wieland LS, Manheimer E, Sampson M, Barnabas JP, Bouter LM, Cho K, Lee MS, Li X, Liu J, Moher D, Okabe T. Bibliometric and content analysis of the Cochrane Complementary Medicine Field specialized register of controlled trials. Systematic reviews. 2013 Dec;2(1):1-3. https://doi.org/10.1186/20464053-2-51

64. Wieland LS, Manheimer E, Berman BM. Development and classification of an operational definition of complementary and alternative medicine for the Cochrane collaboration. Alternative therapies in health and medicine. 2011 Mar;17(2):50. https://www.ncbi.nlm.nih.gov/pmc/articles/PMC3196853/

65. Veziari Y, Leach MJ, Kumar S. Barriers to the conduct and application of research in complementary and alternative medicine: a systematic review. BMC complementary and alternative medicine. 2017 Dec;17(1):1-4. https://doi.org/10.1186/s12906-017-1660-0

\section{Tables}

Table 1: Scopus Search Strategy Executed on April 17, 2021 
ISSN ( 23755776 ) OR ISSN ( 03601293 ) OR ISSN ( 09645284 ) OR ISSN ( 22129588 ) OR ISSN ( 26624052 ) OR ISSN ( 01896016 ) OR ISSN ( 16146891 ) OR ISSN ( 10762809 ) OR ISSN ( 10814000 ) OR ISSN ( $1096942 X$ ) OR ISSN ( 10895159 ) OR ISSN ( 10786791 ) OR ISSN ( 15223396 ) OR ISSN ( 00913960 ) OR ISSN ( 0192415X ) OR ISSN ( 00029157 ) OR ISSN ( 17535174 ) OR ISSN ( 16148339 ) OR ISSN ( 1573420X ) OR ISSN ( 18339735 ) OR ISSN ( 2209119X) OR ISSN ( 10338330 ) OR ISSN ( 10338330 ) OR ISSN ( 14726882 ) OR ISSN ( 07177917 ) OR ISSN ( 00070785 ) OR ISSN ( 16720415 ) OR ISSN ( 20956975 ) OR ISSN ( 17498546 ) OR ISSN ( 02532670 ) OR ISSN ( 09302786 ) OR ISSN ( 2045709X ) OR ISSN ( 17461340 ) OR ISSN ( 10360913 ) OR ISSN ( 08896976 ) OR ISSN ( 14611449 ) OR ISSN ( 15332101 ) OR ISSN ( 02684055 ) OR ISSN ( 25042092 ) OR ISSN ( 17443881 ) OR ISSN ( 09652299 ) OR ISSN ( 13536117 ) OR ISSN ( 22150838 ) OR ISSN ( 04156412 ) OR ISSN ( 18763820 ) OR ISSN ( 13516647 ) OR ISSN ( $1741427 X$ ) OR ISSN ( 11762330 ) OR ISSN ( 15508307 ) OR ISSN ( 14653753 ) OR ISSN ( 16614119 ) OR ISSN ( 00180599 ) OR ISSN ( 08879311 ) OR ISSN ( 14754916 ) OR ISSN ( 09747168 ) OR ISSN ( 09725938 ) OR ISSN ( 15347354 ) OR ISSN ( 1546993X ) OR ISSN ( 23252812 ) OR ISSN ( 11773936 ) OR ISSN ( 22134220 ) OR ISSN ( 19406223 ) OR ISSN ( 09624562 ) OR ISSN ( 10471979 ) OR ISSN ( 19826206 ) OR ISSN ( 17460689 ) OR ISSN ( 19723539 ) OR ISSN ( 09750185 ) OR ISSN ( 20052901 ) OR ISSN ( 16723597 ) OR ISSN ( 10755535 ) OR ISSN ( 10286020 ) OR ISSN ( 09759476 ) OR ISSN ( 22311866 ) OR ISSN ( 13608592 ) OR ISSN ( 15446301 ) OR ISSN ( 01438042 ) OR ISSN ( 15563499 ) OR ISSN ( 15533840 ) OR ISSN ( 14468263 ) OR ISSN ( 17464269 ) OR ISSN ( 21565872 ) OR ISSN ( 13094572 ) OR ISSN ( 12268453 ) OR ISSN ( 22108033 ) OR ISSN ( 10496475 ) OR ISSN ( 19960875 ) OR ISSN ( 16840240 ) OR ISSN ( 18610293 ) OR ISSN ( 01633864 ) OR ISSN ( 08344825 ) OR ISSN ( 20936966 ) OR ISSN ( 10841288 ) OR ISSN ( 13263390 ) OR ISSN ( 1715894 ) OR ISSN ( 22254110 ) OR ISSN ( 18801447 ) OR ISSN ( 18638678 ) OR ISSN ( 00252514 ) OR ISSN ( 19336586 ) OR ISSN ( 11239395 ) OR ISSN ( 15763080 ) OR ISSN ( 07346875 ) OR ISSN ( 1934578X) OR ISSN ( 22103155 ) OR ISSN ( 19408153 ) OR ISSN ( 08098131 ) OR ISSN ( 08039828 ) OR ISSN ( 09747877 ) OR ISSN ( 15982386 ) OR ISSN ( 16159071 ) OR ISSN ( 13880209 ) OR ISSN ( 09737847 ) OR ISSN ( 09580344 ) OR ISSN ( 09723293 ) OR ISSN ( 09447113 ) OR ISSN ( 16248597 ) OR ISSN ( 16286847 ) OR ISSN ( 00320943 ) OR ISSN ( 18193455 ) OR ISSN ( 15160572 ) OR ISSN ( 15760952 ) OR ISSN ( 18878369 ) OR ISSN ( 18888526 ) OR ISSN ( 18789730 ) OR ISSN ( 10150684 ) OR ISSN ( 10950656 ) OR ISSN ( 15564061 ) OR ISSN ( 13021192 ) OR ISSN ( 23264500 ) OR ISSN ( 09735070 ) OR ISSN ( 00986151 ) OR ISSN ( 1560604X) OR ISSN ( 26160684 ) OR ISSN ( 23118571 ) OR ISSN ( 0722348X ) OR ISSN ( 22129596 ) OR ISSN ( 26624060 ) OR ISSN ( 15734218 ) OR ISSN ( 22091203 ) OR ISSN ( 26627671 ) OR ISSN ( 23788763 ) OR ISSN ( 19930402 ) OR ISSN ( 18755364 ) OR ISSN ( 25042106 ) OR ISSN ( 22150846 ) OR ISSN ( 14394359 ) OR ISSN ( 17414288 ) OR ISSN ( 16614127 ) OR ISSN ( 15505138 ) OR ISSN ( 14764245 ) OR ISSN ( 23207094 ) OR ISSN ( 9751068 ) OR ISSN ( 22134239 ) OR ISSN ( 15322106 ) OR ISSN ( 19930399 ) OR ISSN ( 14772213 ) OR ISSN ( 9762809 ) OR ISSN ( 22311874 ) OR ISSN ( 2515690X) OR ISSN ( 21463298 ) OR ISSN ( 20934947 ) OR ISSN ( 22108041 ) OR ISSN ( 15403580 ) OR ISSN ( 21468397 ) OR ISSN ( 15206025 ) OR ISSN ( 22346856 ) OR ISSN ( 20957548 ) OR ISSN ( 18813747 ) OR ISSN ( 14330466 ) OR ISSN ( 15559475 ) OR ISSN ( 22103163 ) OR ISSN ( 22111069 ) OR ISSN ( 17445116 ) OR ISSN ( 9762787 ) OR ISSN ( 10991565 ) OR ISSN ( 17652847 ) OR ISSN ( 14390221 ) OR ISSN ( 19885806 ) OR ISSN ( 23264519 ) OR ISSN ( 26160692 ) OR ISSN ( 10035257 ) OR ISSN ( 25892894 ) OR ISSN ( 16721977 ) OR ISSN ( 10015302 )

Note: The journal titles associated with each ISSN provided in this search strategy is provided in Table 2.

Table 2: Characteristics of CAIM Journals Indexed in Scopus $(n=143)$ 


\begin{tabular}{|c|c|c|c|c|c|c|c|c|}
\hline Position & Journal Name & ISSN & $\begin{array}{l}\text { Active } \\
\text { or } \\
\text { Inactive } \\
\text { (as of } \\
2020 \text { ) }\end{array}$ & $\begin{array}{l}\text { Coverage } \\
\text { Period }\end{array}$ & $\begin{array}{l}\text { Title History } \\
\text { Indication }\end{array}$ & Publisher Name & $\begin{array}{l}\text { Number of } \\
\text { Publications } \\
\text { Published }\end{array}$ & $\begin{array}{l}2019 \\
\text { Impa } \\
\text { Fact }\end{array}$ \\
\hline 1. & $\begin{array}{l}\text { Journal of Natural } \\
\text { Products }\end{array}$ & $\begin{array}{l}1633864 \\
\text { (Print); } \\
15206025 \\
\text { (Electronic) }\end{array}$ & Active & $\begin{array}{l}\text { 1978- } \\
\text { ongoing, } \\
1973,1971, \\
1949\end{array}$ & $\begin{array}{l}\text { Formerly known as } \\
\text { Lloydia }\end{array}$ & $\begin{array}{l}\text { American } \\
\text { Chemical Society }\end{array}$ & 15144 & 3.78: \\
\hline 2. & $\begin{array}{l}\text { Zhongguo Zhongyao } \\
\text { Zazhi }\end{array}$ & $\begin{array}{l}10015302 \\
\text { (Print) }\end{array}$ & Active & $\begin{array}{l}\text { 1989- } \\
\text { ongoing }\end{array}$ & $\begin{array}{l}\text { Formerly known as } \\
\text { Zhong Yao Tong Bao } \\
\text { (Beijing, China: 1981) }\end{array}$ & $\begin{array}{l}\text { Zhongguo Zhongyi } \\
\text { Yanjiuyuan }\end{array}$ & 14577 & N/A \\
\hline 3. & Planta Medica & $\begin{array}{l}00320943 \\
\text { (Print); } \\
14390221 \\
\text { (Electronic) }\end{array}$ & Active & $\begin{array}{l}\text { 1965- } \\
\text { ongoing, } \\
1961\end{array}$ & N/A & $\begin{array}{l}\text { Georg Thieme } \\
\text { Verlag }\end{array}$ & 10793 & $2.68^{\circ}$ \\
\hline 4. & $\begin{array}{l}\text { The Journal of the } \\
\text { American Osteopathic } \\
\text { Association }\end{array}$ & $\begin{array}{l}986151 \\
\text { (Print) }\end{array}$ & Active & $\begin{array}{l}\text { 1945- } \\
\text { ongoing }\end{array}$ & N/A & $\begin{array}{l}\text { American } \\
\text { Osteopathic } \\
\text { Association }\end{array}$ & 9533 & $\mathrm{~N} / \mathrm{A}$ \\
\hline 5. & $\begin{array}{l}\text { Evidence-Based } \\
\text { Complementary and } \\
\text { Alternative Medicine }\end{array}$ & $\begin{array}{l}\text { 1741427X } \\
\text { (Print); } \\
17414288 \\
\text { (Electronic) }\end{array}$ & Active & $\begin{array}{l}\text { 2005- } \\
\text { ongoing }\end{array}$ & $\mathrm{N} / \mathrm{A}$ & $\begin{array}{l}\text { Hindawi Publishing } \\
\text { Corporation }\end{array}$ & 9261 & 1.81: \\
\hline 6. & $\begin{array}{l}\text { Chinese Traditional } \\
\text { and Herbal Drugs }\end{array}$ & $\begin{array}{l}02532670 \\
\text { (Print) }\end{array}$ & Active & $\begin{array}{l}\text { 2006- } \\
\text { ongoing }\end{array}$ & $\mathrm{N} / \mathrm{A}$ & $\begin{array}{l}\text { Chung Tsao Yao } \\
\text { Tsa Chih Pien Chi } \\
\mathrm{Pu}\end{array}$ & 8704 & N/A \\
\hline 7. & $\begin{array}{l}\text { Natural Product } \\
\text { Communications }\end{array}$ & $\begin{array}{l}\text { 1934578X } \\
\text { (Print); } \\
15559475 \\
\text { (Electronic) }\end{array}$ & Active & $\begin{array}{l}\text { 2008- } \\
\text { ongoing }\end{array}$ & $\mathrm{N} / \mathrm{A}$ & $\begin{array}{l}\text { SAGE Publications } \\
\text { Inc. }\end{array}$ & 5800 & $0.46 \uparrow$ \\
\hline 8. & $\begin{array}{l}\text { Pharmaceutical } \\
\text { Biology }\end{array}$ & $\begin{array}{l}13880209 \\
\text { (Print); } \\
17445116 \\
\text { (Electronic) }\end{array}$ & Active & $\begin{array}{l}\text { 1975- } \\
\text { ongoing, } \\
\text { 1961-1972 }\end{array}$ & $\begin{array}{l}\text { Formerly known as } \\
\text { International Journal } \\
\text { of Pharmacognosy }\end{array}$ & Taylor \& Francis & 4901 & 2.97: \\
\hline 9. & Phytomedicine & $\begin{array}{l}09447113 \\
\text { (Print) }\end{array}$ & Active & $\begin{array}{l}\text { 1994- } \\
\text { ongoing }\end{array}$ & N/A & Elsevier BV & 4212 & $4.26\}$ \\
\hline 10. & $\begin{array}{l}\text { BMC Complementary } \\
\text { and Alternative } \\
\text { Medicine }\end{array}$ & $\begin{array}{l}14726882 \\
\text { (Print) }\end{array}$ & Inactive & 2001-2019 & $\begin{array}{l}\text { Continued as BMC } \\
\text { Complementary } \\
\text { Medicine and } \\
\text { Therapies }\end{array}$ & BioMed Central & 3902 & $\begin{array}{l}\mathrm{N} / \mathrm{A} \\
\text { (Inac }\end{array}$ \\
\hline 11. & $\begin{array}{l}\text { Journal of Alternative } \\
\text { and Complementary } \\
\text { Medicine }\end{array}$ & $\begin{array}{l}10755535 \\
\text { (Print) }\end{array}$ & Active & $\begin{array}{l}\text { 1995- } \\
\text { ongoing }\end{array}$ & $\mathrm{N} / \mathrm{A}$ & $\begin{array}{l}\text { Mary Ann Liebert } \\
\text { Inc. }\end{array}$ & 3709 & $2.25 t$ \\
\hline & Journal of Asian & 10286020 & Active & 1998- & $\mathrm{N} / \mathrm{A}$ & Taylor \& Francis & 2822 & $1.34 !$ \\
\hline
\end{tabular}




\begin{tabular}{|c|c|c|c|c|c|c|c|c|}
\hline 12. & $\begin{array}{l}\text { Natural Products } \\
\text { Research }\end{array}$ & $\begin{array}{l}\text { (Print); } \\
14772213 \\
\text { (Electronic) }\end{array}$ & & ongoing & & & & \\
\hline 13. & $\begin{array}{l}\text { American Journal of } \\
\text { Chinese Medicine }\end{array}$ & $\begin{array}{l}\text { 0192415X } \\
\text { (Print) }\end{array}$ & Active & $\begin{array}{l}\text { 1979- } \\
\text { ongoing, } \\
\text { 1974-1977 }\end{array}$ & $\begin{array}{l}\text { Formerly known as } \\
\text { Comparative } \\
\text { Medicine East and } \\
\text { West }\end{array}$ & $\begin{array}{l}\text { World Scientific } \\
\text { Publishing Co }\end{array}$ & 2719 & 3.68: \\
\hline 14. & $\begin{array}{l}\text { British Homeopathic } \\
\text { Journal }\end{array}$ & $\begin{array}{l}00070785 \\
\text { (Print) }\end{array}$ & Inactive & $\begin{array}{l}\text { 1998-2001, } \\
1945-1995\end{array}$ & $\begin{array}{l}\text { Continued as } \\
\text { Homeopathy: The } \\
\text { Journal of the Faculty } \\
\text { of Homeopathy }\end{array}$ & Elsevier BV & 2652 & $\begin{array}{l}\mathrm{N} / \mathrm{A} \\
\text { (Inac }\end{array}$ \\
\hline 15. & $\begin{array}{l}\text { American Journal of } \\
\text { Clinical Hypnosis }\end{array}$ & $\begin{array}{l}00029157 \\
\text { (Print) }\end{array}$ & Active & $\begin{array}{l}\text { 1958- } \\
\text { ongoing }\end{array}$ & $\mathrm{N} / \mathrm{A}$ & $\begin{array}{l}\text { Taylor and Francis } \\
\text { Inc. }\end{array}$ & 2573 & 0.761 \\
\hline 16. & $\begin{array}{l}\text { Complementary } \\
\text { Therapies in Medicine }\end{array}$ & $\begin{array}{l}09652299 \\
\text { (Print) }\end{array}$ & Active & $\begin{array}{l}\text { 1993- } \\
\text { ongoing }\end{array}$ & $\begin{array}{l}\text { Formerly known as } \\
\text { Complementary } \\
\text { Medical Research }\end{array}$ & $\begin{array}{l}\text { Churchill } \\
\text { Livingstone }\end{array}$ & 2436 & 2.06: \\
\hline 17. & $\begin{array}{l}\text { Chinese Journal of } \\
\text { Integrative Medicine }\end{array}$ & $\begin{array}{l}16720415 \\
\text { (Print); } \\
19930402 \\
\text { (Electronic) }\end{array}$ & Active & $\begin{array}{l}\text { 2005- } \\
\text { ongoing }\end{array}$ & $\begin{array}{l}\text { Formerly known as } \\
\text { Chinese Journal of } \\
\text { Integrated Traditional } \\
\text { and Western Medicine }\end{array}$ & $\begin{array}{l}\text { Springer Nature } \\
\text { Switzerland AG }\end{array}$ & 2170 & $1.54 !$ \\
\hline 18. & Manuelle Medizin & $\begin{array}{l}00252514 \\
\text { (Print); } \\
14330466 \\
\text { (Electronic) }\end{array}$ & Active & $\begin{array}{l}\text { 1973- } \\
\text { ongoing }\end{array}$ & N/A & Springer Verlag & 2003 & N/A \\
\hline 19. & $\begin{array}{l}\text { Journal of Bodywork } \\
\text { and Movement } \\
\text { Therapies }\end{array}$ & $\begin{array}{l}13608592 \\
\text { (Print) }\end{array}$ & Active & $\begin{array}{l}\text { 1996- } \\
\text { ongoing }\end{array}$ & N/A & $\begin{array}{l}\text { Churchill } \\
\text { Livingstone }\end{array}$ & 1987 & $\mathrm{~N} / \mathrm{A}$ \\
\hline 20. & $\begin{array}{l}\text { Phytochemical } \\
\text { Analysis }\end{array}$ & $\begin{array}{l}09580344 \\
\text { (Print); } \\
10991565 \\
\text { (Electronic) }\end{array}$ & Active & $\begin{array}{l}\text { 1990- } \\
\text { ongoing }\end{array}$ & $\mathrm{N} / \mathrm{A}$ & $\begin{array}{l}\text { John Wiley \& Sons } \\
\text { Inc. }\end{array}$ & 1954 & 2.77: \\
\hline 21. & $\begin{array}{l}\text { Alternative Therapies } \\
\text { in Health and } \\
\text { Medicine }\end{array}$ & $\begin{array}{l}10786791 \\
\text { (Print) }\end{array}$ & Active & $\begin{array}{l}\text { 1995- } \\
\text { ongoing }\end{array}$ & $\mathrm{N} / \mathrm{A}$ & $\begin{array}{l}\text { InnoVision } \\
\text { Communications }\end{array}$ & 1892 & $0.93^{\circ}$ \\
\hline 22. & $\begin{array}{l}\text { Zhong Xi Yi Jie He Xue } \\
\text { Bao = Journal of } \\
\text { Chinese integrative } \\
\text { medicine }\end{array}$ & $\begin{array}{l}16721977 \\
\text { (Print) }\end{array}$ & Inactive & 2003-2012 & $\begin{array}{l}\text { Continued as Journal } \\
\text { of Integrative } \\
\text { Medicine }\end{array}$ & $\begin{array}{l}\text { Shanghai } \\
\text { Association of } \\
\text { Integrative } \\
\text { Medicine }\end{array}$ & 1861 & $\begin{array}{l}\text { N/A } \\
\text { (Inac }\end{array}$ \\
\hline 23. & $\begin{array}{l}\text { Holistic Nursing } \\
\text { Practice }\end{array}$ & $\begin{array}{l}08879311 \\
\text { (Print); } \\
15505138 \\
\text { (Electronic) }\end{array}$ & Active & $\begin{array}{l}\text { 1986- } \\
\text { ongoing }\end{array}$ & $\begin{array}{l}\text { Formerly known as } \\
\text { Topics in Clinical } \\
\text { Nursing }\end{array}$ & $\begin{array}{l}\text { Lippincott Williams } \\
\text { \& Wilkins Ltd. }\end{array}$ & 1722 & $0.96 \uparrow$ \\
\hline 24. & $\begin{array}{l}\text { Zeitschrift für } \\
\text { Phytotherapie: }\end{array}$ & $\begin{array}{l}\text { 0722348X } \\
\text { (Print) }\end{array}$ & Active & $\begin{array}{l}\text { 1985- } \\
\text { ongoing, }\end{array}$ & $\mathrm{N} / \mathrm{A}$ & $\begin{array}{l}\text { Hippokrates } \\
\text { Verlag }\end{array}$ & 1648 & $\mathrm{~N} / \mathrm{A}$ \\
\hline
\end{tabular}




\begin{tabular}{|c|c|c|c|c|c|c|c|c|}
\hline & $\begin{array}{l}\text { Offizielles Organ der } \\
\text { Ges. f. Phytotherapie } \\
\text { e.V }\end{array}$ & & & 1982 & & & & \\
\hline 25. & $\begin{array}{l}\text { Alternative and } \\
\text { Complementary } \\
\text { Therapies }\end{array}$ & $\begin{array}{l}10762809 \\
\text { (Print) }\end{array}$ & Active & $\begin{array}{l}\text { 1999- } \\
\text { ongoing }\end{array}$ & $\mathrm{N} / \mathrm{A}$ & $\begin{array}{l}\text { Mary Ann Liebert } \\
\text { Inc. }\end{array}$ & 1631 & N/A \\
\hline 26. & $\begin{array}{l}\text { Journal of Medicinal } \\
\text { Plant Research }\end{array}$ & $\begin{array}{l}19960875 \\
\text { (Print) }\end{array}$ & Inactive & 2009-2011 & $\mathrm{N} / \mathrm{A}$ & Academic Journals & 1495 & $\begin{array}{l}\text { N/A } \\
\text { (Inac }\end{array}$ \\
\hline 27. & $\begin{array}{l}\text { EXPLORE: The } \\
\text { Journal of Science } \\
\text { and Healing }\end{array}$ & $\begin{array}{l}15508307 \\
\text { (Print) }\end{array}$ & Active & $\begin{array}{l}\text { 2005- } \\
\text { ongoing }\end{array}$ & $\mathrm{N} / \mathrm{A}$ & Elsevier BV & 1462 & $\overline{1.48 !}$ \\
\hline 28. & $\begin{array}{l}\text { Journal of Natural } \\
\text { Medicines }\end{array}$ & $\begin{array}{l}18610293 \\
\text { (Print) }\end{array}$ & Active & $\begin{array}{l}\text { 2006- } \\
\text { ongoing }\end{array}$ & $\begin{array}{l}\text { Formerly known as } \\
\text { Natural Medicines }\end{array}$ & Springer Verlag & 1461 & $2.05 !$ \\
\hline 29. & $\begin{array}{l}\text { Indian Journal of } \\
\text { Traditional } \\
\text { Knowledge }\end{array}$ & $\begin{array}{l}09725938 \\
\text { (Print); } \\
09751068 \\
\text { (Electronic) }\end{array}$ & Active & $\begin{array}{l}\text { 2008- } \\
\text { ongoing }\end{array}$ & $\mathrm{N} / \mathrm{A}$ & $\begin{array}{l}\text { National Institute } \\
\text { of Science } \\
\text { Communication } \\
\text { and Information } \\
\text { Resources }\end{array}$ & 1446 & $0.73:$ \\
\hline 30. & $\begin{array}{l}\text { Acupuncture in } \\
\text { Medicine }\end{array}$ & $\begin{array}{l}09645284 \\
\text { (Print) }\end{array}$ & Active & $\begin{array}{l}\text { 1996- } \\
\text { ongoing }\end{array}$ & $\mathrm{N} / \mathrm{A}$ & $\begin{array}{l}\text { BMJ Publishing } \\
\text { Group }\end{array}$ & 1391 & $2.12 !$ \\
\hline 31. & $\begin{array}{l}\text { Deutsche Zeitschrift } \\
\text { für Akupunktur }\end{array}$ & $\begin{array}{l}04156412 \\
\text { (Print); } \\
14394359 \\
\text { (Electronic) }\end{array}$ & Inactive & $\begin{array}{l}\text { 1984-2016 } \\
\text { (cancelled) }\end{array}$ & $\mathrm{N} / \mathrm{A}$ & Springer Medizin & 1344 & $\begin{array}{l}\text { N/A } \\
\text { (Inac }\end{array}$ \\
\hline 32. & $\begin{array}{l}\text { Integrative Cancer } \\
\text { Therapies }\end{array}$ & $\begin{array}{l}15347354 \\
\text { (Print) }\end{array}$ & Active & $\begin{array}{l}\text { 2002- } \\
\text { ongoing }\end{array}$ & $\mathrm{N} / \mathrm{A}$ & Sage Science Press & 1258 & $2.37 !$ \\
\hline 33. & $\begin{array}{l}\text { Complementary } \\
\text { Therapies in Clinical } \\
\text { Practice }\end{array}$ & $\begin{array}{l}17443881 \\
\text { (Print) }\end{array}$ & Active & $\begin{array}{l}\text { 2005- } \\
\text { ongoing }\end{array}$ & $\begin{array}{l}\text { Formerly known as } \\
\text { Complementary } \\
\text { Therapies in Nursing } \\
\text { and Midwifery }\end{array}$ & Elsevier BV & 1231 & 1.771 \\
\hline 34. & $\begin{array}{l}\text { Focus on Alternative } \\
\text { and Complementary } \\
\text { Therapies }\end{array}$ & $\begin{array}{l}14653753 \\
\text { (Print) }\end{array}$ & Inactive & $2004-2016$ & $\mathrm{~N} / \mathrm{A}$ & Wiley-Blackwell & 1165 & $\begin{array}{l}\text { N/A } \\
\text { (Inac }\end{array}$ \\
\hline 35. & $\begin{array}{l}\text { Journal of } \\
\text { Acupuncture and } \\
\text { Tuina Science }\end{array}$ & $\begin{array}{l}16723597 \\
\text { (Print); } \\
19930399 \\
\text { (Electronic) }\end{array}$ & Active & $\begin{array}{l}2007- \\
\text { ongoing }\end{array}$ & $\mathrm{N} / \mathrm{A}$ & $\begin{array}{l}\text { Springer Nature } \\
\text { Switzerland AG }\end{array}$ & 1156 & $\mathrm{~N} / \mathrm{A}$ \\
\hline 36. & $\begin{array}{l}\text { Chinese Journal of } \\
\text { Natural Medicines }\end{array}$ & $\begin{array}{l}20956975 \\
\text { (Print); } \\
18755364 \\
\text { (Electronic) }\end{array}$ & Active & $\begin{array}{l}\text { 2004- } \\
\text { ongoing }\end{array}$ & $\mathrm{N} / \mathrm{A}$ & $\begin{array}{l}\text { China } \\
\text { Pharmaceutical } \\
\text { University }\end{array}$ & 1147 & 2.01 \\
\hline
\end{tabular}




\begin{tabular}{|c|c|c|c|c|c|c|c|c|}
\hline 37. & $\begin{array}{l}\text { European Journal of } \\
\text { Integrative Medicine }\end{array}$ & $\begin{array}{l}18763820 \\
\text { (Print) }\end{array}$ & Active & $\begin{array}{l}\text { 2008- } \\
\text { ongoing }\end{array}$ & N/A & Elsevier BV & 1120 & 0.97 \\
\hline 38. & $\begin{array}{l}\text { Revista Brasileira de } \\
\text { Plantas Medicinais }\end{array}$ & $\begin{array}{l}15160572 \\
\text { (Print) }\end{array}$ & Inactive & 1999-2016 & $\mathrm{N} / \mathrm{A}$ & $\begin{array}{l}\text { Fundacao do } \\
\text { Instituto de } \\
\text { Biociencias }\end{array}$ & 1115 & $\begin{array}{l}\text { N/A } \\
\text { (Inac }\end{array}$ \\
\hline 39. & $\begin{array}{l}\text { Journal of Medicinal } \\
\text { Plants }\end{array}$ & $\begin{array}{l}16840240 \\
\text { (Print) }\end{array}$ & Active & $\begin{array}{l}\text { 2004- } \\
\text { ongoing }\end{array}$ & $\mathrm{N} / \mathrm{A}$ & $\begin{array}{l}\text { Pizhuhishkadh-i } \\
\text { giyahan-i darayiva } \\
\text { faravardah ha-vi } \\
\text { tabbii }\end{array}$ & 1090 & N/A \\
\hline 40 & Phytotherapie & $\begin{array}{l}16248597 \\
\text { (Print); } \\
17652847 \\
\text { (Electronic) }\end{array}$ & Active & $\begin{array}{l}\text { 2005- } \\
\text { ongoing }\end{array}$ & N/A & Springer Verlag & 1071 & $\overline{N / A}$ \\
\hline 41 & $\begin{array}{l}\text { African Journal of } \\
\text { Traditional, } \\
\text { Complementary and } \\
\text { Alternative Medicines }\end{array}$ & $\begin{array}{l}01896016 \\
\text { (Print) }\end{array}$ & Inactive & $\begin{array}{l}\text { 2006-2016 } \\
\text { (cancelled), } \\
2002,1995, \\
1991,1981- \\
1982,1973- \\
1978,1970\end{array}$ & $\mathrm{~N} / \mathrm{A}$ & $\begin{array}{l}\text { African Networks } \\
\text { on Ethnomedicines }\end{array}$ & 995 & $\begin{array}{l}\text { N/A } \\
\text { (Inac }\end{array}$ \\
\hline 42. & $\begin{array}{l}\text { Forschende } \\
\text { Komplementarmedizin }\end{array}$ & $\begin{array}{l}16614119 \\
\text { (Print); } \\
16614127 \\
\text { (Electronic) }\end{array}$ & Inactive & $\begin{array}{l}\text { 2006-2016, } \\
2002\end{array}$ & $\begin{array}{l}\text { Formerly known as } \\
\text { Forschende } \\
\text { Komplementarmedizin } \\
\text { und Klassische } \\
\text { Naturheilkunde }\end{array}$ & S. Karger AG & 990 & $\begin{array}{l}\text { N/A } \\
\text { (Inac }\end{array}$ \\
\hline 43. & $\begin{array}{l}\text { Schweizerische } \\
\text { Zeitschrift für } \\
\text { GanzheitsMedizin }\end{array}$ & $\begin{array}{l}10150684 \\
\text { (Print) }\end{array}$ & Inactive & 2002-2017 & $\mathrm{N} / \mathrm{A}$ & $\begin{array}{l}\text { Dr. Becker \& } \\
\text { Partner AG-Verlag } \\
\text { fuer Ganzheits } \\
\text { Medizin }\end{array}$ & 934 & $\begin{array}{l}\text { N/A } \\
\text { (Inac }\end{array}$ \\
\hline 44 & $\begin{array}{l}\text { Homeopathy: The } \\
\text { Journal of the Faculty } \\
\text { of Homeopathy }\end{array}$ & $\begin{array}{l}14754916 \\
\text { (Print); } \\
14764245 \\
\text { (Electronic) }\end{array}$ & Active & $\begin{array}{l}\text { 1998- } \\
\text { ongoing }\end{array}$ & $\begin{array}{l}\text { Formerly known as } \\
\text { British Homeopathic } \\
\text { Journal }\end{array}$ & $\begin{array}{l}\text { Churchill } \\
\text { Livingstone }\end{array}$ & 934 & $\mathrm{~N} / \mathrm{A}$ \\
\hline 45. & $\begin{array}{l}\text { Journal of } \\
\text { Ethnobiology and } \\
\text { Ethnomedicine }\end{array}$ & $\begin{array}{l}17464269 \\
\text { (Print) }\end{array}$ & Active & $\begin{array}{l}\text { 2005- } \\
\text { ongoing }\end{array}$ & $\mathrm{N} / \mathrm{A}$ & BioMed Central & 928 & 2.26 \\
\hline 46. & $\begin{array}{l}\text { Journal of Herbs, } \\
\text { Spices and Medicinal } \\
\text { Plants }\end{array}$ & $\begin{array}{l}10496475 \\
\text { (Print); } \\
15403580 \\
\text { (Electronic) }\end{array}$ & Active & $\begin{array}{l}\text { 1992- } \\
\text { ongoing }\end{array}$ & $\mathrm{N} / \mathrm{A}$ & $\begin{array}{l}\text { The Haworth } \\
\text { Herbal Press }\end{array}$ & 922 & N/A \\
\hline 47. & $\begin{array}{l}\text { American Journal of } \\
\text { Acupuncture }\end{array}$ & $\begin{array}{l}00913960 \\
\text { (Print) }\end{array}$ & Inactive & 1975-1999 & N/A & $\begin{array}{l}\text { American Journal } \\
\text { of Acupuncture }\end{array}$ & 843 & $\begin{array}{l}\mathrm{N} / \mathrm{A} \\
\text { (Inac }\end{array}$ \\
\hline 48. & $\begin{array}{l}\text { Acupuncture and } \\
\text { Electro-Therapeutics }\end{array}$ & $\begin{array}{l}03601293 \\
\text { (Print) }\end{array}$ & Active & $\begin{array}{l}\text { 1976- } \\
\text { ongoing }\end{array}$ & $\mathrm{N} / \mathrm{A}$ & $\begin{array}{l}\text { Cognizant } \\
\text { Communication }\end{array}$ & 838 & 0.201 \\
\hline
\end{tabular}




\begin{tabular}{|c|c|c|c|c|c|c|c|c|}
\hline & Research & & & & & Corporation & & \\
\hline 49. & Medical Acupuncture & $\begin{array}{l}19336586 \\
\text { (Print) }\end{array}$ & Active & $\begin{array}{l}\text { 2008- } \\
\text { ongoing }\end{array}$ & $\mathrm{N} / \mathrm{A}$ & $\begin{array}{l}\text { Mary Ann Liebert } \\
\text { Inc. }\end{array}$ & 825 & N/A \\
\hline 50. & $\begin{array}{l}\text { Journal of Ginseng } \\
\text { Research }\end{array}$ & $\begin{array}{l}12268453 \\
\text { (Print); } \\
20934947 \\
\text { (Electronic) }\end{array}$ & Active & $\begin{array}{l}\text { 2010- } \\
\text { ongoing }\end{array}$ & N/A & Elsevier BV & 770 & $5.48^{\prime}$ \\
\hline 51. & $\begin{array}{l}\text { Boletin } \\
\text { Latinoamericano y del } \\
\text { Caribe de Plantas } \\
\text { Medicinales y } \\
\text { Aromaticas }\end{array}$ & $\begin{array}{l}07177917 \\
\text { (Print) }\end{array}$ & Active & $\begin{array}{l}\text { 2008- } \\
\text { ongoing }\end{array}$ & N/A & $\begin{array}{l}\text { Universidad de } \\
\text { Santiago de Chile }\end{array}$ & 701 & $0.81 !$ \\
\hline 52. & $\begin{array}{l}\text { Journal of } \\
\text { Complementary and } \\
\text { Integrative Medicine }\end{array}$ & $\begin{array}{l}15533840 \\
\text { (Print) }\end{array}$ & Active & $\begin{array}{l}\text { 2006- } \\
\text { ongoing }\end{array}$ & N/A & $\begin{array}{l}\text { Walter de Gruyter } \\
\text { GmbH }\end{array}$ & 699 & N/A \\
\hline 53. & $\begin{array}{l}\text { Journal of Ayurveda } \\
\text { and Integrative } \\
\text { Medicine }\end{array}$ & $\begin{array}{l}09759476 \\
\text { (Print); } \\
09762809 \\
\text { (Electronic) }\end{array}$ & Active & $\begin{array}{l}\text { 2010- } \\
\text { ongoing }\end{array}$ & N/A & Elsevier BV & 690 & N/A \\
\hline 54. & Chinese Medicine & $\begin{array}{l}17498546 \\
\text { (Print) }\end{array}$ & Active & $\begin{array}{l}\text { 2006- } \\
\text { ongoing }\end{array}$ & $\mathrm{N} / \mathrm{A}$ & BioMed Central & 619 & 2.961 \\
\hline 55. & Integrative Medicine & $\begin{array}{l}\text { 1546993X } \\
\text { (Print) }\end{array}$ & Active & $\begin{array}{l}\text { 2013- } \\
\text { ongoing, } \\
\text { 2005-2010 }\end{array}$ & $\begin{array}{l}\text { Formerly known as } \\
\text { International Journal } \\
\text { of Integrative } \\
\text { Medicine }\end{array}$ & $\begin{array}{l}\text { InnoVision } \\
\text { Communications }\end{array}$ & 611 & $\overline{N / A}$ \\
\hline 56. & $\begin{array}{l}\text { Alternative Medicine } \\
\text { Review }\end{array}$ & $\begin{array}{l}10895159 \\
\text { (Print) }\end{array}$ & Inactive & 1996-2012 & $\mathrm{N} / \mathrm{A}$ & $\begin{array}{l}\text { Thorne Reasearch } \\
\text { Inc. }\end{array}$ & 587 & $\begin{array}{l}\mathrm{N} / \mathrm{A} \\
\text { (Inac }\end{array}$ \\
\hline 57. & $\begin{array}{l}\text { Journal of Traditional } \\
\text { and Complementary } \\
\text { Medicine }\end{array}$ & $\begin{array}{l}22254110 \\
\text { (Print) }\end{array}$ & Active & $\begin{array}{l}2011- \\
\text { ongoing }\end{array}$ & N/A & Elsevier BV & 562 & $\overline{N / A}$ \\
\hline 58. & $\begin{array}{l}\text { JAMS Journal of } \\
\text { Acupuncture and } \\
\text { Meridian Studies }\end{array}$ & $\begin{array}{l}20052901 \\
\text { (Print) }\end{array}$ & Active & $\begin{array}{l}\text { 2008- } \\
\text { ongoing }\end{array}$ & N/A & Elsevier BV & 531 & $\overline{N / A}$ \\
\hline 59. & $\begin{array}{l}\text { Chiropractic and } \\
\text { Manual Therapies }\end{array}$ & $\begin{array}{l}\text { 2045709X } \\
\text { (Print) }\end{array}$ & Active & $\begin{array}{l}\text { 2011- } \\
\text { ongoing }\end{array}$ & $\begin{array}{l}\text { Formerly known as } \\
\text { Chiropractic and } \\
\text { Osteopathy }\end{array}$ & BioMed Central & 475 & 1.51: \\
\hline 60. & $\begin{array}{l}\text { Osteopathische } \\
\text { Medizin }\end{array}$ & $\begin{array}{l}16159071 \\
\text { (Print) }\end{array}$ & Inactive & $\begin{array}{l}\text { 2004-2017 } \\
\text { (cancelled) }\end{array}$ & $\mathrm{N} / \mathrm{A}$ & Elsevier BV & 470 & $\begin{array}{l}\mathrm{N} / \mathrm{A} \\
\text { (Inac }\end{array}$ \\
\hline 61. & Medicina Naturista & $\begin{array}{l}15763080 \\
\text { (Print) }\end{array}$ & Active & $\begin{array}{l}2012- \\
\text { ongoing }\end{array}$ & $\mathrm{N} / \mathrm{A}$ & $\begin{array}{l}\text { Zaragoza : } \\
\text { Universidad de } \\
\text { Zaragoza, }\end{array}$ & 462 & N/A \\
\hline
\end{tabular}




\begin{tabular}{|c|c|c|c|c|c|c|c|c|}
\hline & & & & & & $\begin{array}{l}\text { Facultad de } \\
\text { Medicina }\end{array}$ & & \\
\hline 62. & $\begin{array}{l}\text { Journal of Chinese } \\
\text { Medicine }\end{array}$ & $\begin{array}{l}01438042 \\
\text { (Print) }\end{array}$ & Active & $\begin{array}{l}\text { 2016- } \\
\text { ongoing, } \\
\text { 2001-2013 }\end{array}$ & $\mathrm{N} / \mathrm{A}$ & Eastland Press & 459 & $\mathrm{~N} / \mathrm{A}$ \\
\hline 63. & $\begin{array}{l}\text { BMC Complementary } \\
\text { Medicine and } \\
\text { Therapies }\end{array}$ & $\begin{array}{l}26627671 \\
\text { (Electronic) }\end{array}$ & Active & $\begin{array}{l}\text { 2020- } \\
\text { ongoing }\end{array}$ & $\begin{array}{l}\text { Formerly known as } \\
\text { BMC Complementary } \\
\text { and Alternative } \\
\text { Medicine }\end{array}$ & $\begin{array}{l}\text { BioMed Central } \\
\text { Ltd. }\end{array}$ & 456 & $2.83:$ \\
\hline 64. & $\begin{array}{l}\text { International Journal } \\
\text { of Osteopathic } \\
\text { Medicine }\end{array}$ & $\begin{array}{l}17460689 \\
\text { (Print) }\end{array}$ & Active & $\begin{array}{l}\text { 2005- } \\
\text { ongoing }\end{array}$ & $\mathrm{N} / \mathrm{A}$ & Elsevier Ltd & 454 & $\overline{1.20 i}$ \\
\hline 65. & $\begin{array}{l}\text { Revista Internacional } \\
\text { de Acupuntura }\end{array}$ & $\begin{array}{l}18878369 \\
\text { (Print) }\end{array}$ & Active & $\begin{array}{l}2007- \\
\text { ongoing }\end{array}$ & $\mathrm{N} / \mathrm{A}$ & Elsevier BV & 452 & $\overline{N / A}$ \\
\hline 66. & $\begin{array}{l}\text { Tropical Journal of } \\
\text { Natural Product } \\
\text { Research }\end{array}$ & $\begin{array}{l}26160684 \\
\text { (Print); } \\
26160692 \\
\text { (Electronic) }\end{array}$ & Active & $\begin{array}{l}\text { 2017- } \\
\text { ongoing }\end{array}$ & $\mathrm{N} / \mathrm{A}$ & $\begin{array}{l}\text { Faculty of } \\
\text { Pharmacy, } \\
\text { University of Benin }\end{array}$ & 452 & $\overline{N / A}$ \\
\hline 67. & $\begin{array}{l}\text { Nordic Journal of } \\
\text { Music Therapy }\end{array}$ & $\begin{array}{l}08098131 \\
\text { (Print) }\end{array}$ & Active & $\begin{array}{l}\text { 2001- } \\
\text { ongoing }\end{array}$ & $\begin{array}{l}\text { Formerly known as } \\
\text { Nordisk Tidsskrift for } \\
\text { Musikkterapi }\end{array}$ & Taylor \& Francis & 442 & $\overline{0.91:}$ \\
\hline 68. & Chinesische Medizin & $\begin{array}{l}09302786 \\
\text { (Print) }\end{array}$ & Inactive & $\begin{array}{l}\text { 1999-2017 } \\
\text { (cancelled) }\end{array}$ & $\mathrm{N} / \mathrm{A}$ & $\begin{array}{l}\text { Springer } \\
\text { International } \\
\text { Publishing AG }\end{array}$ & 437 & $\begin{array}{l}\mathrm{N} / \mathrm{A} \\
\text { (Inac }\end{array}$ \\
\hline 69. & $\begin{array}{l}\text { International Journal } \\
\text { of Phytomedicine }\end{array}$ & $\begin{array}{l}09750185 \\
\text { (Print) }\end{array}$ & Inactive & $\begin{array}{l}\text { 2010-2016 } \\
\text { (cancelled) }\end{array}$ & $\mathrm{N} / \mathrm{A}$ & $\begin{array}{l}\text { Advanced } \\
\text { Research Journals }\end{array}$ & 427 & $\begin{array}{l}\text { N/A } \\
\text { (Inac }\end{array}$ \\
\hline 70. & Revue d'Homeopathie & $\begin{array}{l}18789730 \\
\text { (Print) }\end{array}$ & Active & $\begin{array}{l}2010- \\
\text { ongoing }\end{array}$ & $\mathrm{N} / \mathrm{A}$ & Elsevier Masson & 416 & $\overline{N / A}$ \\
\hline 71. & $\begin{array}{l}\text { Studies on Ethno- } \\
\text { Medicine }\end{array}$ & $\begin{array}{l}09735070 \\
\text { (Print) }\end{array}$ & Active & $\begin{array}{l}\text { 2009- } \\
\text { ongoing }\end{array}$ & $\mathrm{N} / \mathrm{A}$ & $\begin{array}{l}\text { Kamla-Raj } \\
\text { Enterprises }\end{array}$ & 406 & $\overline{N / A}$ \\
\hline 72. & $\begin{array}{l}\text { Journal of Herbal } \\
\text { Medicine }\end{array}$ & $\begin{array}{l}22108033 \\
\text { (Print); } \\
22108041 \\
\text { (Electronic) }\end{array}$ & Active & $\begin{array}{l}\text { 2011- } \\
\text { ongoing }\end{array}$ & $\mathrm{N} / \mathrm{A}$ & $\begin{array}{l}\text { Urban und Fischer } \\
\text { Verlag Jena }\end{array}$ & 405 & 2.22 \\
\hline 73. & $\begin{array}{l}\text { Journal of Biologically } \\
\text { Active Products from } \\
\text { Nature }\end{array}$ & $\begin{array}{l}22311866 \\
\text { (Print); } \\
22311874 \\
\text { (Electronic) }\end{array}$ & Active & $\begin{array}{l}\text { 2011- } \\
\text { ongoing }\end{array}$ & $\mathrm{N} / \mathrm{A}$ & $\begin{array}{l}\text { Taylor and Francis } \\
\text { Ltd. }\end{array}$ & 402 & $\mathrm{~N} / \mathrm{A}$ \\
\hline 74. & $\begin{array}{l}\text { Natural Products } \\
\text { Journal }\end{array}$ & $\begin{array}{l}22103155 \\
\text { (Print); }\end{array}$ & Active & $\begin{array}{l}\text { 2011- } \\
\text { ongoing }\end{array}$ & $\mathrm{N} / \mathrm{A}$ & $\begin{array}{l}\text { Bentham Science } \\
\text { Publishers B.V. }\end{array}$ & 395 & $\mathrm{~N} / \mathrm{A}$ \\
\hline
\end{tabular}




\begin{tabular}{|c|c|c|c|c|c|c|c|c|}
\hline & & $\begin{array}{l}22103163 \\
\text { (Electronic) }\end{array}$ & & & & & & \\
\hline 75. & $\begin{array}{l}\text { Music Therapy } \\
\text { Perspectives }\end{array}$ & $\begin{array}{l}07346875 \\
\text { (Print) }\end{array}$ & Active & $\begin{array}{l}\text { 2011- } \\
\text { ongoing }\end{array}$ & N/A & $\begin{array}{l}\text { Oxford University } \\
\text { Press }\end{array}$ & 391 & N/A \\
\hline 76. & $\begin{array}{l}\text { Complementary } \\
\text { Therapies in Nursing } \\
\text { and Midwifery }\end{array}$ & $\begin{array}{l}13536117 \\
\text { (Print) }\end{array}$ & Inactive & 1995-2004 & $\begin{array}{l}\text { Continued as } \\
\text { Complementary } \\
\text { Therapies in Clinical } \\
\text { Practice }\end{array}$ & Elsevier BV & 385 & $\begin{array}{l}\text { N/A } \\
\text { (Inac }\end{array}$ \\
\hline 77. & $\begin{array}{l}\text { Complementary } \\
\text { Health Practice } \\
\text { Review }\end{array}$ & $\begin{array}{l}15332101 \\
\text { (Print) }\end{array}$ & Inactive & $\begin{array}{l}\text { 2007-2010, } \\
1996-1999\end{array}$ & $\begin{array}{l}\text { Continued as Journal } \\
\text { of Evidence-Based } \\
\text { Complementary and } \\
\text { Alternative Medicine }\end{array}$ & $\begin{array}{l}\text { SAGE Publications } \\
\text { Inc. }\end{array}$ & 375 & $\begin{array}{l}\mathrm{N} / \mathrm{A} \\
\text { (Inac }\end{array}$ \\
\hline 78. & $\begin{array}{l}\text { Integrative Medicine } \\
\text { Alert }\end{array}$ & $\begin{array}{l}23252812 \\
\text { (Print) }\end{array}$ & Active & $\begin{array}{l}\text { 2013- } \\
\text { ongoing }\end{array}$ & $\begin{array}{l}\text { Formerly known as } \\
\text { Alternative Medicine } \\
\text { Alert }\end{array}$ & $\begin{array}{l}\text { American Health } \\
\text { Consultants, Inc. }\end{array}$ & 374 & $\overline{N / A}$ \\
\hline 79. & $\begin{array}{l}\text { Journal of Evidence- } \\
\text { Based } \\
\text { Complementary and } \\
\text { Alternative Medicine }\end{array}$ & $\begin{array}{l}21565872 \\
\text { (Print) }\end{array}$ & Inactive & $\begin{array}{l}\text { 2014-2018, } \\
1995\end{array}$ & $\begin{array}{l}\text { Continued as Journal } \\
\text { of Evidence-Based } \\
\text { Integrative Medicine }\end{array}$ & SAGE Publications & 369 & $\begin{array}{l}\mathrm{N} / \mathrm{A} \\
\text { (Inac }\end{array}$ \\
\hline 80. & $\begin{array}{l}\text { Oriental Pharmacy } \\
\text { and Experimental } \\
\text { Medicine }\end{array}$ & $\begin{array}{l}15982386 \\
\text { (Print); } \\
22111069 \\
\text { (Electronic) }\end{array}$ & Inactive & $2012-2019$ & $\begin{array}{l}\text { Continued as } \\
\text { Advances in } \\
\text { Traditional Medicine }\end{array}$ & $\begin{array}{l}\text { Springer Science + } \\
\text { Business Media }\end{array}$ & 357 & $\begin{array}{l}\text { N/A } \\
\text { (Inac }\end{array}$ \\
\hline 81. & $\begin{array}{l}\text { KIM - Komplementare } \\
\text { und Integrative } \\
\text { Medizin, } \\
\text { Artztezeitschrift für } \\
\text { Naturheilverfahren }\end{array}$ & $\begin{array}{l}18638678 \\
\text { (Print) }\end{array}$ & Inactive & $2007-2009$ & $\begin{array}{l}\text { Formerly known as } \\
\text { Arztezeitschrift fur } \\
\text { Naturheilverfahren } \\
\text { und } \\
\text { Regulationsmedizin }\end{array}$ & $\begin{array}{l}\text { Urban \& Fischer } \\
\text { Verlag }\end{array}$ & 336 & $\begin{array}{l}\text { N/A } \\
\text { (Inac }\end{array}$ \\
\hline 82. & $\begin{array}{l}\text { Complementary } \\
\text { Medicine Research }\end{array}$ & $\begin{array}{l}25042092 \\
\text { (Print); } \\
25042106 \\
\text { (Electronic) }\end{array}$ & Active & $\begin{array}{l}\text { 2017- } \\
\text { ongoing, } \\
2015\end{array}$ & N/A & S. Karger AG & 314 & 1.08 ! \\
\hline 83. & $\begin{array}{l}\text { Research Journal of } \\
\text { Medicinal Plant }\end{array}$ & $\begin{array}{l}18193455 \\
\text { (Print) }\end{array}$ & Inactive & $\begin{array}{l}\text { 2009-2016 } \\
\text { (cancelled) }\end{array}$ & $\mathrm{N} / \mathrm{A}$ & $\begin{array}{l}\text { Academic Journals } \\
\text { Inc. }\end{array}$ & 311 & $\begin{array}{l}\text { N/A } \\
\text { (Inac }\end{array}$ \\
\hline 84. & $\begin{array}{l}\text { International Journal } \\
\text { of Aromatherapy }\end{array}$ & $\begin{array}{l}09624562 \\
\text { (Print); } \\
15322106 \\
\text { (Electronic) }\end{array}$ & Inactive & 1995-2006 & $\mathrm{N} / \mathrm{A}$ & $\begin{array}{l}\text { Essential Oil } \\
\text { Resource } \\
\text { Consultants }\end{array}$ & 307 & $\begin{array}{l}\text { N/A } \\
\text { (Inac }\end{array}$ \\
\hline 85. & $\begin{array}{l}\text { International Journal } \\
\text { of High Dilution } \\
\text { Research }\end{array}$ & $\begin{array}{l}19826206 \\
\text { (Print) }\end{array}$ & Active & $\begin{array}{l}\text { 2011- } \\
\text { ongoing }\end{array}$ & $\mathrm{N} / \mathrm{A}$ & $\begin{array}{l}\text { Universidade } \\
\text { Estadual Paulista - } \\
\text { UNESP }\end{array}$ & 300 & $\mathrm{~N} / \mathrm{A}$ \\
\hline 86. & Herba Polonica & $\begin{array}{l}00180599 \\
\text { (Print) }\end{array}$ & Active & $\begin{array}{l}\text { 2018- } \\
\text { ongoing, }\end{array}$ & $\mathrm{N} / \mathrm{A}$ & $\begin{array}{l}\text { Instytut Roslin i } \\
\text { Przetworow }\end{array}$ & 284 & $\mathrm{~N} / \mathrm{A}$ \\
\hline
\end{tabular}




\begin{tabular}{|c|c|c|c|c|c|c|c|c|}
\hline & & & & 1973-1979 & & Zielarskich & & \\
\hline 87. & Sleep and Hypnosis & $\begin{array}{l}13021192 \\
\text { (Print) }\end{array}$ & Active & $\begin{array}{l}\text { 2000- } \\
\text { ongoing }\end{array}$ & $\mathrm{N} / \mathrm{A}$ & $\begin{array}{l}\text { Kure Iletisim } \\
\text { Grubu A S }\end{array}$ & 282 & $\sqrt[N / A]{ }$ \\
\hline 88. & $\begin{array}{l}\text { Thermology } \\
\text { International }\end{array}$ & $\begin{array}{l}\text { 1560604X } \\
\text { (Print) }\end{array}$ & Active & $\begin{array}{l}\text { 2002- } \\
\text { ongoing }\end{array}$ & $\mathrm{N} / \mathrm{A}$ & $\begin{array}{l}\text { European } \\
\text { Association of } \\
\text { Thermology }\end{array}$ & 278 & N/A \\
\hline 89. & $\begin{array}{l}\text { Journal of the } \\
\text { Australian } \\
\text { Traditional-Medicine } \\
\text { Society }\end{array}$ & $\begin{array}{l}13263390 \\
\text { (Print) }\end{array}$ & Inactive & 2008-2016 & $\mathrm{N} / \mathrm{A}$ & \begin{tabular}{|l} 
Australian \\
Traditional- \\
Medicine Society
\end{tabular} & 276 & $\begin{array}{l}\mathrm{N} / \mathrm{A} \\
\text { (Inac }\end{array}$ \\
\hline 90. & $\begin{array}{l}\text { Journal of Traditional } \\
\text { Medicines }\end{array}$ & $\begin{array}{l}18801447 \\
\text { (Print); } \\
18813747 \\
\text { (Electronic) }\end{array}$ & Inactive & $2004-2013$ & $\mathrm{~N} / \mathrm{A}$ & $\begin{array}{l}\text { Medical and } \\
\text { Pharmaceutical } \\
\text { Society for } \\
\text { WAKAN-YAKU }\end{array}$ & 268 & $\begin{array}{l}\text { N/A } \\
\text { (Inac }\end{array}$ \\
\hline 91. & $\begin{array}{l}\text { Arztezeitschrift für } \\
\text { Naturheilverfahren } \\
\text { und } \\
\text { Regulationsmedizin }\end{array}$ & $\begin{array}{l}16148339 \\
\text { (Print) }\end{array}$ & Inactive & $2004-2006$ & $\begin{array}{l}\text { Continued as KIM - } \\
\text { Komplementare und } \\
\text { Integrative Medizin, } \\
\text { Artztezeitschrift fur } \\
\text { Naturheilverfahren }\end{array}$ & $\begin{array}{l}\text { Medizinisch } \\
\text { Literarische } \\
\text { Verlagsgesellschaft } \\
\text { mbH }\end{array}$ & 266 & $\begin{array}{l}\text { N/A } \\
\text { (Inac }\end{array}$ \\
\hline 92. & $\begin{array}{l}\text { Revista Medica de } \\
\text { Homeopatia }\end{array}$ & $\begin{array}{l}18888526 \\
\text { (Print) }\end{array}$ & Inactive & $2008-2017$ & $\mathrm{~N} / \mathrm{A}$ & Elsevier Doyma & 261 & $\begin{array}{l}\mathrm{N} / \mathrm{A} \\
\text { (Inac }\end{array}$ \\
\hline 93. & $\begin{array}{l}\text { Pharmacognosy } \\
\text { Reviews }\end{array}$ & $\begin{array}{l}09737847 \\
\text { (Print); } \\
09762787 \\
\text { (Electronic) }\end{array}$ & Inactive & $\begin{array}{l}\text { 2009-2018 } \\
\text { (cancelled) }\end{array}$ & $\mathrm{N} / \mathrm{A}$ & $\begin{array}{l}\text { Medknow } \\
\text { Publications }\end{array}$ & 255 & $\begin{array}{l}\text { N/A } \\
\text { (Inac }\end{array}$ \\
\hline 94. & $\begin{array}{l}\text { Advances in } \\
\text { Integrative Medicine }\end{array}$ & $\begin{array}{l}22129588 \\
\text { (Print); } \\
22129596 \\
\text { (Electronic) }\end{array}$ & Active & $\begin{array}{l}\text { 2013- } \\
\text { ongoing }\end{array}$ & $\mathrm{N} / \mathrm{A}$ & Elsevier BV & 254 & $\mathrm{~N} / \mathrm{A}$ \\
\hline 95. & $\begin{array}{l}\text { Journal of } \\
\text { Chiropractic } \\
\text { Humanities }\end{array}$ & $\begin{array}{l}15563499 \\
\text { (Print) }\end{array}$ & Active & $\begin{array}{l}\text { 2010- } \\
\text { ongoing }\end{array}$ & $\mathrm{N} / \mathrm{A}$ & Elsevier BV & 247 & $\mathrm{~N} / \mathrm{A}$ \\
\hline 96. & Asian Medicine & $\begin{array}{l}\text { 1573420X } \\
\text { (Print); } \\
15734218 \\
\text { (Electronic) } \\
\end{array}$ & Active & $\begin{array}{l}\text { 2007- } \\
\text { ongoing }\end{array}$ & $\mathrm{N} / \mathrm{A}$ & Brill & 241 & N/A \\
\hline 97. & Natural Solutions & $\begin{array}{l}19408153 \\
\text { (Print) }\end{array}$ & Inactive & $\begin{array}{l}\text { 2009-2011, } \\
1996-1997\end{array}$ & $\mathrm{~N} / \mathrm{A}$ & $\begin{array}{l}\text { Alternative } \\
\text { Medicine.com }\end{array}$ & 226 & $\begin{array}{l}\mathrm{N} / \mathrm{A} \\
\text { (Inac }\end{array}$ \\
\hline 98. & $\begin{array}{l}\text { Spirituality in Clinical } \\
\text { Practice }\end{array}$ & $\begin{array}{l}23264500 \\
\text { (Print); } \\
23264519 \\
\text { (Electronic) }\end{array}$ & Active & $\begin{array}{l}\text { 2014- } \\
\text { ongoing }\end{array}$ & $\mathrm{N} / \mathrm{A}$ & $\begin{array}{l}\text { American } \\
\text { Psychological } \\
\text { Association Inc. }\end{array}$ & 222 & N/A \\
\hline
\end{tabular}




\begin{tabular}{|c|c|c|c|c|c|c|c|c|}
\hline 99. & $\begin{array}{l}\text { Alternative Medicine } \\
\text { Alert }\end{array}$ & $\begin{array}{l}1096942 \mathrm{X} \\
\text { (Print) }\end{array}$ & Inactive & 2009-2012 & $\begin{array}{l}\text { Continued as } \\
\text { Integrative Medicine } \\
\text { Alert }\end{array}$ & $\begin{array}{l}\text { American Health } \\
\text { Consultants, Inc. }\end{array}$ & 220 & $\begin{array}{l}\text { N/A } \\
\text { (Inac }\end{array}$ \\
\hline 100. & $\begin{array}{l}\text { Journal of } \\
\text { Complementary } \\
\text { Medicine }\end{array}$ & $\begin{array}{l}14468263 \\
\text { (Print) }\end{array}$ & Inactive & 2008-2009 & $\mathrm{N} / \mathrm{A}$ & $\begin{array}{l}\text { Australian } \\
\text { Pharmaceutical } \\
\text { Publishing Co., } \\
\text { Ltd. }\end{array}$ & 205 & $\begin{array}{l}\text { N/A } \\
\text { (Inac }\end{array}$ \\
\hline 101. & $\begin{array}{l}\text { World Journal of } \\
\text { Traditional Chinese } \\
\text { Medicine }\end{array}$ & $\begin{array}{l}23118571 \\
\text { (Print); } \\
25892894 \\
\text { (Electronic) } \\
\end{array}$ & Active & $\begin{array}{l}2017- \\
\text { ongoing }\end{array}$ & $\mathrm{N} / \mathrm{A}$ & $\begin{array}{l}\text { Wolters Kluwer } \\
\text { Medknow } \\
\text { Publications }\end{array}$ & 200 & $\mathrm{~N} / \mathrm{A}$ \\
\hline 102. & $\begin{array}{l}\text { Australian Journal of } \\
\text { Medical Herbalism }\end{array}$ & $\begin{array}{l}10338330 \\
\text { (Print) }\end{array}$ & Inactive & 2006-2017 & $\begin{array}{l}\text { Continued as } \\
\text { Australian Journal of } \\
\text { Herbal and } \\
\text { Naturopathic } \\
\text { Medicine }\end{array}$ & $\begin{array}{l}\text { National } \\
\text { Herbalists } \\
\text { Association of } \\
\text { Australia }\end{array}$ & 177 & $\begin{array}{l}\text { N/A } \\
\text { (Inac }\end{array}$ \\
\hline 103. & $\begin{array}{l}\text { International Journal } \\
\text { of Applied Research } \\
\text { in Natural Products }\end{array}$ & $\begin{array}{l}19406223 \\
\text { (Print) }\end{array}$ & Inactive & $\begin{array}{l}\text { 2008-2016 } \\
\text { (cancelled) }\end{array}$ & $\mathrm{N} / \mathrm{A}$ & $\begin{array}{l}\text { Healthy Synergies } \\
\text { Publications }\end{array}$ & 176 & $\begin{array}{l}\mathrm{N} / \mathrm{A} \\
\text { (Inac }\end{array}$ \\
\hline 104. & $\begin{array}{l}\text { Nordisk Tidsskrift for } \\
\text { Musikkterapi }\end{array}$ & $\begin{array}{l}08039828 \\
\text { (Print) }\end{array}$ & Inactive & $1992-2000$ & $\begin{array}{l}\text { Continued as Nordic } \\
\text { Journal of Music } \\
\text { Therapy }\end{array}$ & $\begin{array}{l}\text { Taylor and Francis } \\
\text { Ltd. }\end{array}$ & 175 & $\begin{array}{l}\mathrm{N} / \mathrm{A} \\
\text { (Inac }\end{array}$ \\
\hline 105. & $\begin{array}{l}\text { Journal of } \\
\text { Pharmacopuncture }\end{array}$ & $\begin{array}{l}20936966 \\
\text { (Print); } \\
22346856 \\
\text { (Electronic) } \\
\end{array}$ & Active & $\begin{array}{l}\text { 2016- } \\
\text { ongoing }\end{array}$ & $\mathrm{N} / \mathrm{A}$ & $\begin{array}{l}\text { Korean } \\
\text { Pharmacopuncture } \\
\text { Institute }\end{array}$ & 173 & $\mathrm{~N} / \mathrm{A}$ \\
\hline 106. & $\begin{array}{l}\text { European Journal of } \\
\text { Oriental Medicine }\end{array}$ & $\begin{array}{l}13516647 \\
\text { (Print) }\end{array}$ & Inactive & \begin{tabular}{|l|}
$2017,2006-$ \\
2014
\end{tabular} & $\mathrm{~N} / \mathrm{A}$ & $\begin{array}{l}\text { British } \\
\text { Acupuncture } \\
\text { Council }\end{array}$ & 159 & $\begin{array}{l}\text { N/A } \\
\text { (Inac }\end{array}$ \\
\hline 107. & Phytomedica & $\begin{array}{l}09723293 \\
\text { (Print) }\end{array}$ & Inactive & 1999-2006 & $\begin{array}{l}\text { Formerly known as } \\
\text { Indian Journal of } \\
\text { Indigenous Medicines }\end{array}$ & $\begin{array}{l}\text { Scientific } \\
\text { Publishers }\end{array}$ & 158 & $\begin{array}{l}\mathrm{N} / \mathrm{A} \\
\text { (Inac }\end{array}$ \\
\hline 108. & $\begin{array}{l}\text { Advances in } \\
\text { Traditional Medicine }\end{array}$ & $\begin{array}{l}26624052 \\
\text { (Print); } \\
26624060 \\
\text { (Electronic) } \\
\end{array}$ & Active & $\begin{array}{l}\text { 2020- } \\
\text { ongoing }\end{array}$ & $\begin{array}{l}\text { Formerly known as } \\
\text { Oriental Pharmacy } \\
\text { and Experimental } \\
\text { Medicine }\end{array}$ & $\begin{array}{l}\text { Springer } \\
\text { Singapore }\end{array}$ & 157 & $\mathrm{~N} / \mathrm{A}$ \\
\hline 109. & $\begin{array}{l}\text { Chiropractic Journal } \\
\text { of Australia }\end{array}$ & $\begin{array}{l}10360913 \\
\text { (Print) }\end{array}$ & Active & $\begin{array}{l}2016- \\
\text { ongoing, } \\
2010-2013\end{array}$ & $\mathrm{~N} / \mathrm{A}$ & $\begin{array}{l}\text { Chiropractors' } \\
\text { Association of } \\
\text { Australia }\end{array}$ & 151 & $\mathrm{~N} / \mathrm{A}$ \\
\hline 110. & $\begin{array}{l}\text { Journal of Sports } \\
\text { Chiropractic and } \\
\text { Rehabilitation }\end{array}$ & $\begin{array}{l}10841288 \\
\text { (Print) }\end{array}$ & Inactive & $1996-2001$ & $\begin{array}{l}\text { Formerly known as } \\
\text { Chiropractic Sports } \\
\text { Medicine }\end{array}$ & Atwood Publishing & 147 & $\begin{array}{l}\text { N/A } \\
\text { (Inac }\end{array}$ \\
\hline 111. & $\begin{array}{l}\text { Cannabis and } \\
\text { Cannabinoid } \\
\text { Research }\end{array}$ & $\begin{array}{l}23788763 \\
\text { (Electronic) }\end{array}$ & Active & $\begin{array}{l}\text { 2016- } \\
\text { ongoing }\end{array}$ & $\mathrm{N} / \mathrm{A}$ & $\begin{array}{l}\text { Mary Ann Liebert } \\
\text { Inc. }\end{array}$ & 147 & $\mathrm{~N} / \mathrm{A}$ \\
\hline
\end{tabular}




\begin{tabular}{|c|c|c|c|c|c|c|c|c|}
\hline 112. & Revista de Fitoterapia & $\begin{array}{l}15760952 \\
\text { (Print); } \\
19885806 \\
\text { (Electronic) }\end{array}$ & Active & \begin{tabular}{|l}
$2009-$ \\
ongoing
\end{tabular} & $\mathrm{N} / \mathrm{A}$ & $\begin{array}{l}\text { Cita Publicaciones } \\
\text { y Documentacion } \\
\text { SL }\end{array}$ & 144 & $\mathrm{~N} / \mathrm{A}$ \\
\hline 113. & $\begin{array}{l}\text { Chiropractic and } \\
\text { Osteopathy }\end{array}$ & $\begin{array}{l}17461340 \\
\text { (Print) }\end{array}$ & Inactive & $2005-2010$ & $\begin{array}{l}\text { Continued as } \\
\text { Chiropractic and } \\
\text { Manual Therapies }\end{array}$ & BioMed Central & 135 & $\begin{array}{l}\text { N/A } \\
\text { (Inac }\end{array}$ \\
\hline 114. & $\begin{array}{l}\text { Scientific Review of } \\
\text { Alternative Medicine }\end{array}$ & $\begin{array}{l}10950656 \\
\text { (Print) }\end{array}$ & Inactive & 2000-2007 & N/A & $\begin{array}{l}\text { Prometheus Books } \\
\text { Inc. }\end{array}$ & 134 & $\begin{array}{l}\mathrm{N} / \mathrm{A} \\
\text { (Inac }\end{array}$ \\
\hline 115. & $\begin{array}{l}\text { Chiropractic Sports } \\
\text { Medicine }\end{array}$ & $\begin{array}{l}08896976 \\
\text { (Print) }\end{array}$ & Inactive & 1987-1995 & $\begin{array}{l}\text { Continued as Journal } \\
\text { of Sports Chiropractic } \\
\text { and Rehabilitation }\end{array}$ & Atwood Publishing & 133 & $\begin{array}{l}\mathrm{N} / \mathrm{A} \\
\text { (Inac }\end{array}$ \\
\hline 116. & $\begin{array}{l}\text { Clinical Acupuncture } \\
\text { and Oriental Medicine }\end{array}$ & $\begin{array}{l}14611449 \\
\text { (Print) }\end{array}$ & Inactive & 1999-2003 & $\mathrm{N} / \mathrm{A}$ & $\begin{array}{l}\text { Churchill } \\
\text { Livingstone }\end{array}$ & 133 & $\begin{array}{l}\mathrm{N} / \mathrm{A} \\
\text { (Inac }\end{array}$ \\
\hline 117. & $\begin{array}{l}\text { International Journal } \\
\text { of Ozone Therapy }\end{array}$ & $\begin{array}{l}19723539 \\
\text { (Print) }\end{array}$ & Inactive & 2007-2013 & $\mathrm{N} / \mathrm{A}$ & Centauro SRL & 129 & $\begin{array}{l}\mathrm{N} / \mathrm{A} \\
\text { (Inac }\end{array}$ \\
\hline 118. & $\begin{array}{l}\text { Journal of the Society } \\
\text { for Integrative } \\
\text { Oncology }\end{array}$ & $\begin{array}{l}1715894 X \\
\text { (Print) }\end{array}$ & Inactive & 2006-2010 & $\begin{array}{l}\text { Formerly known as } \\
\text { Journal of Cancer } \\
\text { Integrative Medicine }\end{array}$ & B.C. Decker Inc. & 128 & $\begin{array}{l}\mathrm{N} / \mathrm{A} \\
\text { (Inac }\end{array}$ \\
\hline 119. & $\begin{array}{l}\text { Journal of } \\
\text { Intercultural } \\
\text { Ethnopharmacology }\end{array}$ & $\begin{array}{l}21468397 \\
\text { (Electronic) }\end{array}$ & Inactive & $\begin{array}{l}\text { 2016-2017 } \\
\text { (cancelled) }\end{array}$ & N/A & Ejmanager LLC & 122 & $\begin{array}{l}\mathrm{N} / \mathrm{A} \\
\text { (Inac }\end{array}$ \\
\hline 120. & $\begin{array}{l}\text { Medicina Clinica e } \\
\text { Termale }\end{array}$ & $\begin{array}{l}11239395 \\
\text { (Print) }\end{array}$ & Inactive & $\begin{array}{l}2008,2005- \\
2006,2001- \\
2003,1997- \\
1998\end{array}$ & $\mathrm{~N} / \mathrm{A}$ & $\begin{array}{l}\text { Tipografia la } \\
\text { Commerciale s.n.c. }\end{array}$ & 122 & $\begin{array}{l}\mathrm{N} / \mathrm{A} \\
\text { (Inac }\end{array}$ \\
\hline 121. & $\begin{array}{l}\text { Phytotherapie } \\
\text { Europeenne }\end{array}$ & $\begin{array}{l}16286847 \\
\text { (Print) }\end{array}$ & Inactive & $2007-2010$ & $\mathrm{~N} / \mathrm{A}$ & Meditions Carline & 121 & $\begin{array}{l}\mathrm{N} / \mathrm{A} \\
\text { (Inac }\end{array}$ \\
\hline 122. & Alternative Medicine & $\begin{array}{l}10814000 \\
\text { (Print) }\end{array}$ & Inactive & $2007-2008$ & $\mathrm{~N} / \mathrm{A}$ & $\begin{array}{l}\text { Future Medicine } \\
\text { Pub. }\end{array}$ & 119 & $\begin{array}{l}\mathrm{N} / \mathrm{A} \\
\text { (Inac }\end{array}$ \\
\hline 123. & $\begin{array}{l}\text { Complementary } \\
\text { Medical Research }\end{array}$ & $\begin{array}{l}02684055 \\
\text { (Print) }\end{array}$ & Inactive & 1988-1992 & $\begin{array}{l}\text { Continued as } \\
\text { Complementary } \\
\text { Therapies in Medicine }\end{array}$ & $\begin{array}{l}\text { Routledge \& Kegan } \\
\text { Paul }\end{array}$ & 119 & $\begin{array}{l}\mathrm{N} / \mathrm{A} \\
\text { (Inac }\end{array}$ \\
\hline 124. & $\begin{array}{l}\text { Journal of Traditional } \\
\text { Chinese Medical } \\
\text { Sciences }\end{array}$ & $\begin{array}{l}20957548 \\
\text { (Electronic) }\end{array}$ & Active & $\begin{array}{l}\text { 2019- } \\
\text { ongoing }\end{array}$ & $\mathrm{N} / \mathrm{A}$ & $\begin{array}{l}\text { Beijing University } \\
\text { of Chinese } \\
\text { Medicine }\end{array}$ & 115 & $\mathrm{~N} / \mathrm{A}$ \\
\hline 125. & $\begin{array}{l}\text { Journal of Evidence- } \\
\text { Based Integrative } \\
\text { Medicine }\end{array}$ & $\begin{array}{l}2515690 X \\
\text { (Electronic) }\end{array}$ & Active & $\begin{array}{l}\text { 2018- } \\
\text { ongoing }\end{array}$ & $\begin{array}{l}\text { Formerly known as } \\
\text { Journal of Evidence- } \\
\text { Based }\end{array}$ & $\begin{array}{l}\text { SAGE Publications } \\
\text { Ltd }\end{array}$ & 113 & $\mathrm{~N} / \mathrm{A}$ \\
\hline
\end{tabular}




\begin{tabular}{|c|c|c|c|c|c|c|c|c|}
\hline & & & & & $\begin{array}{l}\text { Complementary and } \\
\text { Alternative Medicine }\end{array}$ & & & \\
\hline 126. & $\begin{array}{l}\text { Alternative Therapies } \\
\text { in Womens Health }\end{array}$ & $\begin{array}{l}15223396 \\
\text { (Print) }\end{array}$ & Inactive & 2006-2009 & $\mathrm{N} / \mathrm{A}$ & $\begin{array}{l}\text { American Health } \\
\text { Consultant }\end{array}$ & 97 & $\begin{array}{l}\mathrm{N} / \mathrm{A} \\
\text { (Inac }\end{array}$ \\
\hline 127. & $\begin{array}{l}\text { Integrative Medicine } \\
\text { Research }\end{array}$ & $\begin{array}{l}22134220 \\
\text { (Print); } \\
22134239 \\
\text { (Electronic) }\end{array}$ & Active & $\begin{array}{l}\text { 2020- } \\
\text { ongoing }\end{array}$ & N/A & Elsevier BV & 96 & 2.17: \\
\hline 128. & $\begin{array}{l}\text { Australian Journal of } \\
\text { Acupuncture and } \\
\text { Chinese Medicine }\end{array}$ & $\begin{array}{l}18339735 \\
\text { (Print) }\end{array}$ & Ina ctive & $\begin{array}{l}\text { 2016-2016, } \\
2011-2014\end{array}$ & $\mathrm{~N} / \mathrm{A}$ & $\begin{array}{l}\text { Australian } \\
\text { Acupuncture and } \\
\text { Chinese Medicine } \\
\text { Association Ltd }\end{array}$ & 83 & $\begin{array}{l}\mathrm{N} / \mathrm{A} \\
\text { (Inac }\end{array}$ \\
\hline 129. & AAO Journal & $\begin{array}{l}23755776 \\
\text { (Print) }\end{array}$ & Active & $\begin{array}{l}\text { 2006- } \\
\text { ongoing }\end{array}$ & $\mathrm{N} / \mathrm{A}$ & $\begin{array}{l}\text { American Academy } \\
\text { Of Osteopathy }\end{array}$ & 83 & $\mathrm{~N} / \mathrm{A}$ \\
\hline 130. & $\begin{array}{l}\text { Australian Journal of } \\
\text { Herbal and } \\
\text { Naturopathic } \\
\text { Medicine }\end{array}$ & $\begin{array}{l}\text { 2209119X } \\
\text { (Print); } \\
22091203 \\
\text { (Electronic) }\end{array}$ & Active & $\begin{array}{l}\text { 2018- } \\
\text { ongoing }\end{array}$ & $\begin{array}{l}\text { Formerly known as } \\
\text { Australian Journal of } \\
\text { Medical Herbalism }\end{array}$ & $\begin{array}{l}\text { Naturopaths and } \\
\text { Herbalists } \\
\text { Association of } \\
\text { Australia }\end{array}$ & 63 & N/A \\
\hline 131. & $\begin{array}{l}\text { Indian Journal of } \\
\text { Research in } \\
\text { Homoeopathy }\end{array}$ & $\begin{array}{l}09747168 \\
\text { (Print); } \\
23207094 \\
\text { (Electronic) }\end{array}$ & Active & $\begin{array}{l}\text { 2019- } \\
\text { ongoing }\end{array}$ & N/A & $\begin{array}{l}\text { Wolters Kluwer } \\
\text { Medknow } \\
\text { Publications }\end{array}$ & 61 & $\mathrm{~N} / \mathrm{A}$ \\
\hline 132. & $\begin{array}{l}\text { Akupunktur und } \\
\text { Traditionelle } \\
\text { Chinesische Medizin }\end{array}$ & $\begin{array}{l}16146891 \\
\text { (Print) }\end{array}$ & Inactive & 2004-2006 & $\begin{array}{l}\text { Formerly known as } \\
\text { Akupunktur }\end{array}$ & $\begin{array}{l}\text { Medizinisch } \\
\text { Literarische } \\
\text { Verlagsgesellschaft } \\
\text { mbH }\end{array}$ & 59 & $\begin{array}{l}\text { N/A } \\
\text { (Inac }\end{array}$ \\
\hline 133. & $\begin{array}{l}\text { Seminars in } \\
\text { Preventive and } \\
\text { Alternative Medicine }\end{array}$ & $\begin{array}{l}15564061 \\
\text { (Print) }\end{array}$ & Inactive & $2005-2007$ & $\mathrm{~N} / \mathrm{A}$ & Elsevier & 43 & $\begin{array}{l}\text { N/A } \\
\text { (Inac }\end{array}$ \\
\hline 134. & $\begin{array}{l}\text { Open Access Journal } \\
\text { of Medicinal and } \\
\text { Aromatic Plants }\end{array}$ & $\begin{array}{l}09747877 \\
\text { (Print) }\end{array}$ & Active & $\begin{array}{l}\text { 2010- } \\
\text { ongoing }\end{array}$ & $\mathrm{N} / \mathrm{A}$ & $\begin{array}{l}\text { Medicinal and } \\
\text { Aromatic Plants } \\
\text { Association of } \\
\text { India }\end{array}$ & 41 & $\mathrm{~N} / \mathrm{A}$ \\
\hline 135. & $\begin{array}{l}\text { Archives of Drug } \\
\text { Information }\end{array}$ & $\begin{array}{l}17535174 \\
\text { (Print) }\end{array}$ & Inactive & 2008-2011 & $\mathrm{N} / \mathrm{A}$ & $\begin{array}{l}\text { John Wiley \& Sons } \\
\text { Inc. }\end{array}$ & 35 & $\begin{array}{l}\text { N/A } \\
\text { (Inac }\end{array}$ \\
\hline 136. & $\begin{array}{l}\text { Integrative Medicine } \\
\text { Insights }\end{array}$ & $\begin{array}{l}11773936 \\
\text { (Print) }\end{array}$ & Inactive & $2008-2018$ & $\begin{array}{l}\text { Continued as Journal } \\
\text { of Evidence-Based } \\
\text { Integrative Medicine }\end{array}$ & \begin{tabular}{|l} 
Libertas \\
Academica
\end{tabular} & 33 & $\begin{array}{l}\text { N/A } \\
\text { (Inac }\end{array}$ \\
\hline 137. & $\begin{array}{l}\text { Evidence-Based } \\
\text { Integrative Medicine }\end{array}$ & $\begin{array}{l}11762330 \\
\text { (Print) }\end{array}$ & Inactive & 2005 & $\mathrm{~N} / \mathrm{A}$ & Adis Press & 31 & $\begin{array}{l}\mathrm{N} / \mathrm{A} \\
\text { (Inac }\end{array}$ \\
\hline & Current Traditional & 22150838 & Inactive & $2015-2016$ & $\mathrm{~N} / \mathrm{A}$ & Bentham Science & 16 & $\overline{N / A}$ \\
\hline
\end{tabular}




\begin{tabular}{|c|c|c|c|c|c|c|c|c|}
\hline 138. & Medicine & $\begin{array}{l}\text { (Print); } \\
22150846 \\
\text { (Electronic) }\end{array}$ & & & & Publishers B.V. & & (Inac \\
\hline 139. & $\begin{array}{l}\text { Journal of } \\
\text { Experimental and } \\
\text { Integrative Medicine }\end{array}$ & $\begin{array}{l}13094572 \\
\text { (Print); } \\
21463298 \\
\text { (Electronic) }\end{array}$ & Inactive & $2014-2016$ & $\mathrm{~N} / \mathrm{A}$ & Gesdav & 16 & $\begin{array}{l}\mathrm{N} / \mathrm{A} \\
\text { (Inac }\end{array}$ \\
\hline 140. & $\begin{array}{l}\text { Journal of Cancer } \\
\text { Integrative Medicine }\end{array}$ & $\begin{array}{l}15446301 \\
\text { (Print) }\end{array}$ & Inactive & 2005 & $\begin{array}{l}\text { Continued as Journal } \\
\text { of the Society for } \\
\text { Integrative Oncology }\end{array}$ & $\begin{array}{l}\text { Prime National } \\
\text { Publishing Corp. }\end{array}$ & 15 & $\begin{array}{l}\mathrm{N} / \mathrm{A} \\
\text { (Inac }\end{array}$ \\
\hline 141. & $\begin{array}{l}\text { International Journal } \\
\text { of Clinical } \\
\text { Acupuncture }\end{array}$ & $\begin{array}{l}10471979 \\
\text { (Print) }\end{array}$ & Inactive & 2014-2016 & $\mathrm{N} / \mathrm{A}$ & Allerton Press Inc. & 4 & $\begin{array}{l}\text { N/A } \\
\text { (Inac }\end{array}$ \\
\hline 142. & $\begin{array}{l}\text { World Journal of } \\
\text { Acupuncture - } \\
\text { Moxibustion }\end{array}$ & $\begin{array}{l}10035257 \\
\text { (Electronic) }\end{array}$ & Inactive & 2013 & $\mathrm{~N} / \mathrm{A}$ & Elsevier BV & 4 & $\begin{array}{l}\text { N/A } \\
\text { (Inac }\end{array}$ \\
\hline 143. & $\begin{array}{l}\text { Journal of } \\
\text { Orthomolecular } \\
\text { Medicine }\end{array}$ & $\begin{array}{l}08344825 \\
\text { (Print) }\end{array}$ & Inactive & $\begin{array}{l}2017,1988- \\
2015\end{array}$ & $\begin{array}{l}\text { Formerly known as } \\
\text { Journal of } \\
\text { Orthomolecular } \\
\text { Psychiatry }\end{array}$ & \begin{tabular}{|l} 
Canadian \\
Schizophrenia \\
Foundation
\end{tabular} & 0 & $\begin{array}{l}\mathrm{N} / \mathrm{A} \\
\text { (Inac }\end{array}$ \\
\hline
\end{tabular}

Table 3: General Characteristics of Publications in CAIM Journals 
Publication Volume

Number of Total Publications $(\mathrm{n}=172466)$

Number of Open Access Publications $(n=42331)$

Document Type (\# of

publications)

\begin{tabular}{|l} 
Article $(\mathrm{n}=139540)$ \\
\hline Review $(\mathrm{n}=13418)$ \\
\hline Note $(\mathrm{n}=5873)$ \\
\hline Editorial $(\mathrm{n}=4643)$ \\
\hline Letter $(\mathrm{n}=3096)$ \\
\hline Conference Paper $(\mathrm{n}=2706)$ \\
\hline Short Survey $(\mathrm{n}=1774)$ \\
\hline Erratum $(\mathrm{n}=1293)$ \\
\hline Retracted $(\mathrm{n}=23)$ \\
\hline Conference Review $(\mathrm{n}=5)$ \\
\hline Undefined $(\mathrm{n}=95)$ \\
\hline
\end{tabular}

Number of Unique Authors $(n=219680)$

Source Titles (Journals) Across All Publications $(\mathrm{n}=144)$

Subject Area of Publication (10 Highest)

(\# of publications)

Medicine $(\mathrm{n}=172456)$

Pharmacology, Toxicology and Pharmaceutics $(\mathrm{n}=86902)$

Biochemistry, Genetics and Molecular Biology $(n=40262)$

Chemistry $(\mathrm{n}=31845)$

Agricultural and Biological Sciences $(n=12124)$

Health Professions ( $\mathrm{n}=11487)$

Nursing $(\mathrm{n}=7428)$

Social Sciences $(n=1949)$

Mathematics $(\mathrm{n}=1461)$

Arts and Humanities $(\mathrm{n}=1249)$

Language of Publication (10 Highest)

(\# of publications)

English $(\mathrm{n}=135718)$

Chinese $(n=24614)$

German $(\mathrm{n}=8611)$

Spanish $(\mathrm{n}=1741)$

French $(\mathrm{n}=1645)$

Portuguese $(\mathrm{n}=1065)$

Persian $(\mathrm{n}=691)$

Polish $(\mathrm{n}=200)$

Italian $(\mathrm{n}=162)$

Arabic $(\mathrm{n}=120)$

Country of Publication (20 Highest)

(\# of publications)

China $(\mathrm{n}=45860)$

United States $(\mathrm{n}=29523)$

Germany $(\mathrm{n}=10120)$

India $(\mathrm{n}=9116)$

Japan $(\mathrm{n}=6774)$ 


\begin{tabular}{|l|} 
South Korea $(\mathrm{n}=6120)$ \\
\hline United Kingdom $(\mathrm{n}=6057)$ \\
\hline Brazil $(\mathrm{n}=4915)$ \\
\hline Australia $(\mathrm{n}=3844)$ \\
\hline Taiwan $(\mathrm{n}=3660)$ \\
\hline Iran $(\mathrm{n}=3558)$ \\
\hline Italy $(\mathrm{n}=3341)$ \\
\hline France $(\mathrm{n}=3325)$ \\
\hline Canada $(\mathrm{n}=2607)$ \\
\hline Spain $(\mathrm{n}=2449)$ \\
\hline Switzerland $(\mathrm{n}=2238)$ \\
\hline Austria $(\mathrm{n}=1835)$ \\
\hline Nigeria $(\mathrm{n}=1814)$ \\
\hline Malaysia $(\mathrm{n}=1798)$ \\
\hline Turkey $(\mathrm{n}=1754)$ \\
\hline
\end{tabular}

Institutional Affiliation (20 Highest)

(\# of publications)

China Academy of Chinese Medical Sciences $(\mathrm{n}=3560)$

Beijing University of Chinese Medicine $(n=2896)$

Chinese Academy of Sciences $(n=2896)$

Chinese Academy of Medical Sciences \& Peking Union Medical College ( $\mathrm{n}=2613)$

Ministry of Education China $(\mathrm{n}=2595)$

Shanghai University of Traditional Chinese Medicine $(\mathrm{n}=1949)$

Nanjing University of Traditional Chinese Medicine ( $\mathrm{n}=1868)$

China Pharmaceutical University $(n=1403)$

Kyung Hee University ( $\mathrm{n}=1302$ )

Tianjin University of Traditional Chinese Medicine $(n=1171)$

China Medical University Taichung ( $\mathrm{n}=1165)$

Chengdu University of Traditional Chinese Medicine $(n=1153)$

Shenyang Pharmaceutical University $(n=1147)$

Guangzhou University of Chinese Medicine (n=1078)

Institute of Materia Medica, Chinese Academy of Medical Sciences \& Peking Union Medical College

$(\mathrm{n}=1069)$

Institute of Medicinal Plant Development, Chinese Academy of Medical Sciences \& Peking Union

Medical College $(n=1035)$

Peking University $(\mathrm{n}=963)$

Kunming Institute of Botany Chinese Academy of Sciences $(\mathrm{n}=941)$

Jiangxi University of Traditional Chinese Medicine $(n=853)$

Korea Institute of Oriental Medicine $(\mathrm{n}=785)$

Funding Sponsor (20 Highest)

(\# of publications)

National Natural Science Foundation of China $(n=5711)$

National Institutes of Health $(n=4055)$

US Department of Health and Human Services $(n=4032)$

National Cancer Institute $(\mathrm{n}=1477)$

Ministry of Education, Culture, Sports, Science and Technology (n=1057) 
Ministry of Science and Technology of the People's Republic of China ( $\mathrm{n}=979)$

National Research Foundation of Korea $(\mathrm{n}=944)$

National Center for Complementary and Integrative Health $(\mathrm{n}=932)$

Japan Society for the Promotion of Science $(n=903)$

Ministry of Education of the People's Republic of China $(\mathrm{n}=749)$

Conselho Nacional de Desenvolvimento Científico e Tecnológico (n=694)

Ministério da Ciência, Tecnologia e Inovações (n=574)

Coordenação de Aperfeicoamento de Pessoal de Nível Superior $(n=527)$

National Institute of General Medical Sciences $(n=442)$

National Center for Research Resources $(n=427)$

National Key Research and Development Program of China $(\mathrm{n}=397)$

Ministry of Science, ICT and Future Planning $(\mathrm{n}=353)$

Fundamental Research Funds for the Central Universities $(n=323)$

European Commission ( $\mathrm{n}=309)$

Chinese Academy of Sciences $(n=293)$

Table 4: 100 Most Productive Authors Across Publications in CAIM Journals 


\begin{tabular}{|c|c|c|}
\hline Position & Author Name & Number of Publications \\
\hline 1. & Huang, L.Q. & 330 \\
\hline 2. & Goetz, P. & 314 \\
\hline 3. & Ernst, E. & 311 \\
\hline 4. & Xie, Y.M. & 264 \\
\hline 5. & Lee, M.S. & 232 \\
\hline 6. & Tu, P.F. & 231 \\
\hline 7. & Pezzuto, J.M. & 223 \\
\hline 8. & Jia, X.B. & 213 \\
\hline 9. & Kinghorn, A.D. & 207 \\
\hline 10. & Cordell, G.A. & 205 \\
\hline 11. & Guo, L.P. & 186 \\
\hline 12. & Yang, M. & 186 \\
\hline 13. & Dossey, L. & 179 \\
\hline 14. & Wang, Z.Z. & 176 \\
\hline & Kingston, D.G.I. & 170 \\
\hline
\end{tabular}

Page 25/43 


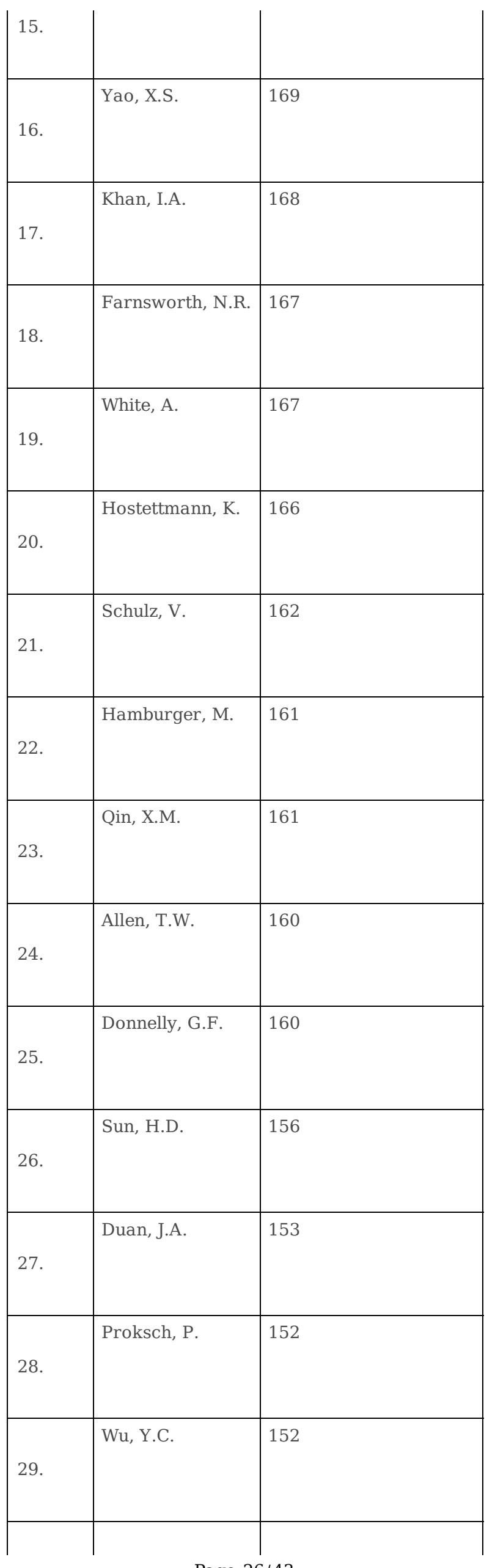

Page 26/43 


\begin{tabular}{|c|c|c|}
\hline 30. & Guo, Q.S. & 151 \\
\hline 31. & Setzer, W.N. & 146 \\
\hline 32. & Adams, J. & 145 \\
\hline 33. & Efferth, T. & 145 \\
\hline 34. & Ammer, $\mathrm{K}$. & 140 \\
\hline 35. & Ye, W.C. & 140 \\
\hline 36. & Zhang, T.J. & 140 \\
\hline 37. & Bauer, R. & 137 \\
\hline 38. & Yang, S.L. & 136 \\
\hline 39. & Hart, J. & 134 \\
\hline 40 & Lee, K.H. & 134 \\
\hline 41. & Sticher, O. & 134 \\
\hline 42. & Yarnell, E. & 134 \\
\hline 43. & Pettit, G.R. & 133 \\
\hline 44. & Uehleke, B. & 133 \\
\hline
\end{tabular}

Page 27/43 


\begin{tabular}{|c|c|c|}
\hline 45. & Yang, X.W. & 131 \\
\hline 46. & Chen, S.L. & 130 \\
\hline 47. & Wang, Z.M. & 128 \\
\hline 48. & Xiao, X.H. & 127 \\
\hline 49. & Lin, J.G. & 126 \\
\hline 50. & Beyer, L. & 124 \\
\hline 51. & Kiefer, D. & 124 \\
\hline 52. & Choudhary, M.I. & 122 \\
\hline 53. & Pieters, L. & 122 \\
\hline 54. & Chen, R.Y. & 120 \\
\hline 55. & Robinson, N. & 120 \\
\hline 56. & Verpoorte, R. & 119 \\
\hline 57. & Liao, $\mathrm{X}$. & 118 \\
\hline 58. & Zhang, W.D. & 116 \\
\hline 59. & Hao, X.J. & 115 \\
\hline
\end{tabular}

Page 28/43 


\begin{tabular}{|c|c|c|}
\hline 60. & Block, K.I. & 114 \\
\hline 61. & Kraft, K. & 114 \\
\hline 62. & Liebenson, C. & 114 \\
\hline 63. & Kadota, S. & 112 \\
\hline 64. & Lao, L. & 112 \\
\hline 65. & Litscher, G. & 112 \\
\hline 66. & Wagner, H. & 112 \\
\hline 67. & Niemtzow, R.C. & 111 \\
\hline 68. & Yu, D.Q. & 111 \\
\hline 69. & Fisher, P. & 110 \\
\hline 70. & Kong, L.Y. & 110 \\
\hline 71. & Omura, Y. & 110 \\
\hline 72. & McLaughlin, J.L. & 108 \\
\hline 73. & Tezuka, Y. & 108 \\
\hline & Chang, F.R. & 107 \\
\hline
\end{tabular}

Page 29/43 


\begin{tabular}{|c|c|c|}
\hline 74. & & \\
\hline 75. & Fong, H.H.S. & 106 \\
\hline 76. & Gibson, D.M. & 106 \\
\hline 77. & Cramer, H. & 105 \\
\hline 78. & Yuan, Y. & 105 \\
\hline 79. & Horowitz, S. & 104 \\
\hline 80. & Walach, H. & 104 \\
\hline 81. & Gerwick, W.H. & 103 \\
\hline 82. & Wright, A.D. & 103 \\
\hline 83. & Naghdi Badi, H. & 102 \\
\hline 84. & Qian, D.W. & 102 \\
\hline 85. & Kuo, Y.H. & 101 \\
\hline 86. & Schwartz, S.A. & 101 \\
\hline 87. & Dai, H.F. & 100 \\
\hline 88. & Guo, D.A. & 100 \\
\hline & & \\
\hline
\end{tabular}

Page 30/43 


\begin{tabular}{|c|c|c|}
\hline 89. & Hsieh, C.L. & 100 \\
\hline 90. & Xiao, P.G. & 100 \\
\hline 91. & Li, P. & 99 \\
\hline 92. & Morita, H. & 99 \\
\hline 93. & De Tommasi, N. & 98 \\
\hline 94. & Ots, T. & 98 \\
\hline 95. & Tokuda, H. & 97 \\
\hline 96. & Weeks, J. & 97 \\
\hline 97. & Witt, C.M. & 97 \\
\hline 98. & Saller, R. & 96 \\
\hline 99. & Steel, A. & 96 \\
\hline 100. & König, G.M. & 95 (tied for $100^{\text {th }}$ place) \\
\hline 101. & Xiao, W. & 95 (tied for $100^{\text {th }}$ place) \\
\hline
\end{tabular}




\begin{tabular}{|c|c|c|c|c|c|}
\hline Position & Title & Authors & Year & Source Title & $\begin{array}{l}\text { Citation } \\
\text { Count }\end{array}$ \\
\hline 1. & $\begin{array}{l}\text { Natural products as } \\
\text { sources of new drugs } \\
\text { over the last } 25 \text { years }\end{array}$ & Newman D.J., Cragg G.M. & 2007 & $\begin{array}{l}\text { Journal of } \\
\text { Natural } \\
\text { Products }\end{array}$ & 3258 \\
\hline 2. & $\begin{array}{l}\text { Flavonoids as } \\
\text { antioxidants }\end{array}$ & Pietta P.-G. & 2000 & $\begin{array}{l}\text { Journal of } \\
\text { Natural } \\
\text { Products }\end{array}$ & 3162 \\
\hline 3. & $\begin{array}{l}\text { Natural products as } \\
\text { sources of new drugs } \\
\text { over the } 30 \text { years from } \\
1981 \text { to } 2010\end{array}$ & Newman D.J., Cragg G.M. & 2012 & $\begin{array}{l}\text { Journal of } \\
\text { Natural } \\
\text { Products }\end{array}$ & 3122 \\
\hline 4. & $\begin{array}{l}\text { Brine shrimp: A } \\
\text { convenient general } \\
\text { bioassay for active plant } \\
\text { constituents }\end{array}$ & $\begin{array}{l}\text { Meyer B.N., Ferrigni N.R., Putnam J.E., Jacobsen L.B., Nichols } \\
\text { D.E., McLaughlin J.L. }\end{array}$ & 1982 & Planta Medica & 2939 \\
\hline 5. & $\begin{array}{l}\text { Natural Products as } \\
\text { Sources of New Drugs } \\
\text { from } 1981 \text { to } 2014\end{array}$ & Newman D.J., Cragg G.M. & 2016 & $\begin{array}{l}\text { Journal of } \\
\text { Natural } \\
\text { Products }\end{array}$ & 2750 \\
\hline 6. & $\begin{array}{l}\text { Natural products as } \\
\text { sources of new drugs } \\
\text { over the period 1981- } \\
2002\end{array}$ & Newman D.J., Cragg G.M., Snader K.M. & 2003 & $\begin{array}{l}\text { Journal of } \\
\text { Natural } \\
\text { Products }\end{array}$ & 2285 \\
\hline 7. & $\begin{array}{l}\text { Free radicals, } \\
\text { antioxidants and } \\
\text { functional foods: Impact } \\
\text { on human health }\end{array}$ & Lobo V., Patil A., Phatak A., Chandra N. & 2010 & $\begin{array}{l}\text { Pharmacognosy } \\
\text { Reviews }\end{array}$ & 1939 \\
\hline 8. & $\begin{array}{l}\text { Pharmacology of } \\
\text { Curcuma longa }\end{array}$ & Ammon H.P.T., Wahl M.A. & 1991 & Planta Medica & 1415 \\
\hline 9. & $\begin{array}{l}\text { A sensitive and quick } \\
\text { microplate method to } \\
\text { determine the minimal } \\
\text { inhibitory concentration } \\
\text { of plant extracts for } \\
\text { bacteria }\end{array}$ & Eloff J.N. & 1998 & Planta Medica & 1320 \\
\hline 10. & $\begin{array}{l}\text { Screening of plant } \\
\text { extracts for antioxidant } \\
\text { activity: A comparative } \\
\text { study on three testing } \\
\text { methods }\end{array}$ & $\begin{array}{l}\text { Koleva I.I., Van Beek T.A., Linssen J.P.H., De Groot A., } \\
\text { Evstatieva L.N. }\end{array}$ & 2002 & $\begin{array}{l}\text { Phytochemical } \\
\text { Analysis }\end{array}$ & 1186 \\
\hline 11. & $\begin{array}{l}\text { Influence of piperine on } \\
\text { the pharmacokinetics of } \\
\text { curcumin in animals and } \\
\text { human volunteers }\end{array}$ & $\begin{array}{l}\text { Shoba G., Joy D., Joseph T., Majeed M., Rajendran R., Srinivas } \\
\text { P.S.S.R. }\end{array}$ & 1998 & Planta Medica & 1178 \\
\hline
\end{tabular}




\begin{tabular}{|c|c|c|c|c|c|}
\hline 12. & $\begin{array}{l}\text { Natural products in drug } \\
\text { discovery and } \\
\text { development }\end{array}$ & Cragg G.M., Newman D.J., Snader K.M. & 1997 & \begin{tabular}{|l} 
Journal of \\
Natural \\
Products
\end{tabular} & 1126 \\
\hline 13. & $\begin{array}{l}\text { Natural polyphenols } \\
\text { (vegetable tannins) as } \\
\text { drugs: Possible modes of } \\
\text { action }\end{array}$ & Haslam E. & 1996 & $\begin{array}{l}\text { Journal of } \\
\text { Natural } \\
\text { Products }\end{array}$ & 960 \\
\hline 14. & $\begin{array}{l}\text { Preferred reporting items } \\
\text { for systematic reviews } \\
\text { and meta-analyses: The } \\
\text { PRISMA statement } \\
\text { (Chinese edition) }\end{array}$ & $\begin{array}{l}\text { Moher D., Liberati A., Tetzlaff J., Altman D.G., Altman D., } \\
\text { Antes G., Atkins D., Barbour V., Barrowman N., Berlin J.A., } \\
\text { Clark J., Clarke M., Cook D., D'Amico R., Deeks J.J., Devereaux } \\
\text { P.J., Dickersin K., Egger M., Ernst E., Gøtzsche P.C., } \\
\text { Grimshaw J., Guyatt G., Higgins J., Ioannidis J.P.A., Kleijnen J., } \\
\text { Lang T., Magrini N., McNamee D., Moja L., Mulrow C., Napoli } \\
\text { M., Oxman A., Pham B., Rennie D., Sampson M., Schulz K.F., } \\
\text { Shekelle P.G., Tovey D., Tugwell P. }\end{array}$ & 2009 & $\begin{array}{l}\text { Journal of } \\
\text { Chinese } \\
\text { Integrative } \\
\text { Medicine }\end{array}$ & 933 \\
\hline 15. & $\begin{array}{l}\text { The role of natural } \\
\text { product chemistry in } \\
\text { drug discovery }\end{array}$ & Butler M.S. & 2004 & $\begin{array}{l}\text { Journal of } \\
\text { Natural } \\
\text { Products }\end{array}$ & 918 \\
\hline 16. & $\begin{array}{l}\text { Natural Products from } \\
\text { Endophytic } \\
\text { Microorganisms }\end{array}$ & Strobel G., Daisy B., Castillo U., Harper J. & 2004 & $\begin{array}{l}\text { Journal of } \\
\text { Natural } \\
\text { Products }\end{array}$ & 916 \\
\hline 17. & $\begin{array}{l}\text { Anti-inflammatory } \\
\text { properties of curcumin, a } \\
\text { major constituent of } \\
\text { Curcuma longa: A review } \\
\text { of preclinical and clinical } \\
\text { research }\end{array}$ & Jurenka J.S. & 2009 & $\begin{array}{l}\text { Alternative } \\
\text { Medicine } \\
\text { Review }\end{array}$ & 837 \\
\hline 18. & $\begin{array}{l}\text { Dose escalation of a } \\
\text { curcuminoid formulation }\end{array}$ & $\begin{array}{l}\text { Lao C.D., Ruffin IV M.T., Normolle D., Heath D.D., Murray } \\
\text { S.I., Bailey J.M., Boggs M.E., Crowell J., Rock C.L., Brenner } \\
\text { D.E. }\end{array}$ & 2006 & $\begin{array}{l}\text { BMC } \\
\text { Complementary } \\
\text { and Alternative } \\
\text { Medicine }\end{array}$ & 833 \\
\hline 19. & $\begin{array}{l}\text { Structure-activity } \\
\text { relationship and } \\
\text { classification of } \\
\text { flavonoids as inhibitors } \\
\text { of xanthine oxidase and } \\
\text { superoxide scavengers }\end{array}$ & $\begin{array}{l}\text { Cos P., Ying L., Calomme M., Hu J.P., Cimanga K., Van Poel B., } \\
\text { Pieters L., Vlietinck A.J., Vanden Berghe D. }\end{array}$ & 1998 & $\begin{array}{l}\text { Journal of } \\
\text { Natural } \\
\text { Products }\end{array}$ & 823 \\
\hline 20. & $\begin{array}{l}\text { Mindfulness-based stress } \\
\text { reduction for stress } \\
\text { management in healthy } \\
\text { people: A review and } \\
\text { meta-analysis }\end{array}$ & Chiesa A., Serretti A. & 2009 & $\begin{array}{l}\text { Journal of } \\
\text { Alternative and } \\
\text { Complementary } \\
\text { Medicine }\end{array}$ & 789 \\
\hline 21. & $\begin{array}{l}\text { Th1/Th2 balance: The } \\
\text { hypothesis, its } \\
\text { limitations, and }\end{array}$ & Kidd P. & 2003 & $\begin{array}{l}\text { Alternative } \\
\text { Medicine } \\
\text { Review }\end{array}$ & 781 \\
\hline
\end{tabular}




\begin{tabular}{|c|c|c|c|c|c|}
\hline & $\begin{array}{l}\text { implications for health } \\
\text { and disease }\end{array}$ & & & & \\
\hline 22. & $\begin{array}{l}\text { Annonaceous } \\
\text { acetogenins: Recent } \\
\text { progress }\end{array}$ & Alali F.Q., Liu X.-X., McLaughlin J.L. & 1999 & $\begin{array}{l}\text { Journal of } \\
\text { Natural } \\
\text { Products }\end{array}$ & 745 \\
\hline 23. & $\begin{array}{l}\text { Antidiabetic plants and } \\
\text { their active constituents }\end{array}$ & Marles R.J., Farnsworth N.R. & 1995 & Phytomedicine & 744 \\
\hline 24. & $\begin{array}{l}\text { Trends in use of } \\
\text { complementary and } \\
\text { alternative medicine by } \\
\text { us adults: } 1997-2002\end{array}$ & Tindle H.A., Davis R.B., Phillips R.S., Eisenberg D.M. & 2005 & $\begin{array}{l}\text { Alternative } \\
\text { Therapies in } \\
\text { Health and } \\
\text { Medicine }\end{array}$ & 724 \\
\hline 25. & $\begin{array}{l}\text { Antioxidant principles } \\
\text { from Bauhinia } \\
\text { tarapotensis }\end{array}$ & $\begin{array}{l}\text { Braca A., De Tommasi N., Di Bari L., Pizza C., Politi M., } \\
\text { Morelli I. }\end{array}$ & 2001 & $\begin{array}{l}\text { Journal of } \\
\text { Natural } \\
\text { Products }\end{array}$ & 712 \\
\hline 26. & $\begin{array}{l}\text { Synergy research: } \\
\text { Approaching a new } \\
\text { generation of } \\
\text { phytopharmaceuticals }\end{array}$ & Wagner H., Ulrich-Merzenich G. & 2009 & Phytomedicine & 695 \\
\hline 27. & $\begin{array}{l}\text { Safety and anti- } \\
\text { inflammatory activity of } \\
\text { curcumin: A component } \\
\text { of tumeric (Curcuma } \\
\text { longa) }\end{array}$ & Chainani-Wu N. & 2003 & $\begin{array}{l}\text { Journal of } \\
\text { Alternative and } \\
\text { Complementary } \\
\text { Medicine }\end{array}$ & 683 \\
\hline 28. & $\begin{array}{l}\text { Natural products from } \\
\text { plant-associated } \\
\text { microorganisms: } \\
\text { Distribution, structural } \\
\text { diversity, bioactivity, and } \\
\text { implications of their } \\
\text { occurrence }\end{array}$ & Gunatilaka A.A.L. & 2006 & $\begin{array}{l}\text { Journal of } \\
\text { Natural } \\
\text { Products }\end{array}$ & 664 \\
\hline 29. & $\begin{array}{l}\text { The pharmacological } \\
\text { potential of mushrooms }\end{array}$ & Lindequist U., Niedermeyer T.H.J., Jülich W.-D. & 2005 & $\begin{array}{l}\text { Evidence-based } \\
\text { Complementary } \\
\text { and Alternative } \\
\text { Medicine }\end{array}$ & 648 \\
\hline 30. & $\begin{array}{l}\text { Fixed oil of Nigella sativa } \\
\text { and derived } \\
\text { thymoquinone inhibit } \\
\text { eicosanoid generation in } \\
\text { leukocytes and } \\
\text { membrane lipid } \\
\text { peroxidation }\end{array}$ & Houghton P.J., Zarka R., De Las Heras B., Hoult J.R.S. & 1995 & Planta Medica & 641 \\
\hline 31. & $\begin{array}{l}\text { Marine natural products } \\
\text { and related compounds }\end{array}$ & Newman D.J., Cragg G.M. & 2004 & $\begin{array}{l}\text { Journal of } \\
\text { Natural } \\
\text { Products }\end{array}$ & 626 \\
\hline
\end{tabular}




\begin{tabular}{|c|c|c|c|c|c|}
\hline & $\begin{array}{l}\text { in clinical and advanced } \\
\text { preclinical trials }\end{array}$ & & & & \\
\hline 32. & $\begin{array}{l}\text { Synergy and other } \\
\text { interactions in } \\
\text { phytomedicines }\end{array}$ & Williamson E.M. & 2001 & Phytomedicine & 621 \\
\hline 33. & $\begin{array}{l}\text { Nrf2 as a master redox } \\
\text { switch in turning on the } \\
\text { cellular signaling } \\
\text { involved in the induction } \\
\text { of cytoprotective genes } \\
\text { by some chemopreventive } \\
\text { phytochemicals }\end{array}$ & Surh Y.-J., Kundu J.K., Na H.-K. & 2008 & Planta Medica & 593 \\
\hline 34. & $\begin{array}{l}\text { Use and expenditure on } \\
\text { complementary medicine } \\
\text { in England: A population } \\
\text { based survey }\end{array}$ & Thomas K.J., Nicholl J.P., Coleman P. & 2001 & $\begin{array}{l}\text { Complementary } \\
\text { Therapies in } \\
\text { Medicine }\end{array}$ & 577 \\
\hline 35. & $\begin{array}{l}\text { In vitro antibacterial } \\
\text { activity of some plant } \\
\text { essential oils }\end{array}$ & Prabuseenivasan S., Jayakumar M., Ignacimuthu S. & 2006 & $\begin{array}{l}\text { BMC } \\
\text { Complementary } \\
\text { and Alternative } \\
\text { Medicine }\end{array}$ & 572 \\
\hline 36. & $\begin{array}{l}\text { Antioxidant and } \\
\text { antiinflammatory } \\
\text { activities of anthocyanins } \\
\text { and their aglycon, } \\
\text { cyanidin, from tart } \\
\text { cherries }\end{array}$ & $\begin{array}{l}\text { Wang H., Nair M.G., Strasburg G.M., Chang Y.-C., Booren } \\
\text { A.M., Gray J.I., DeWitt D.L. }\end{array}$ & 1999 & $\begin{array}{l}\text { Journal of } \\
\text { Natural } \\
\text { Products }\end{array}$ & 572 \\
\hline 37. & $\begin{array}{l}\text { Alkaloids from } \\
\text { amphibian skin: A } \\
\text { tabulation of over eight- } \\
\text { hundred compounds }\end{array}$ & Daly J.W., Spande T.F., Garraffo H.M. & 2005 & $\begin{array}{l}\text { Journal of } \\
\text { Natural } \\
\text { Products }\end{array}$ & 558 \\
\hline 38. & $\begin{array}{l}\text { Scientific basis for the } \\
\text { therapeutic use of } \\
\text { Withania somnifera } \\
\text { (ashwagandha): A review }\end{array}$ & Mishra L.-C., Singh B.B., Dagenais S. & 2000 & $\begin{array}{l}\text { Alternative } \\
\text { Medicine } \\
\text { Review }\end{array}$ & 538 \\
\hline 39. & $\begin{array}{l}\text { Acetylcholinesterase } \\
\text { inhibitors from plants }\end{array}$ & Mukherjee P.K., Kumar V., Mal M., Houghton P.J. & 2007 & Phytomedicine & 522 \\
\hline 40 & $\begin{array}{l}\text { Extraction, isolation and } \\
\text { characterization of } \\
\text { bioactive compounds } \\
\text { from plants' extracts }\end{array}$ & $\begin{array}{l}\text { Sasidharan S., Chen Y., Saravanan D., Sundram K.M., Yoga } \\
\text { Latha L. }\end{array}$ & 2011 & $\begin{array}{l}\text { African Journal } \\
\text { of Traditional, } \\
\text { Complementary } \\
\text { and Alternative } \\
\text { Medicines }\end{array}$ & 514 \\
\hline 41. & $\begin{array}{l}\text { Annonaceous } \\
\text { acetogenins: A review }\end{array}$ & Rupprecht J.K., Hui Y.-H., McLaughlin J.L. & 1990 & $\begin{array}{l}\text { Journal of } \\
\text { Natural } \\
\text { Products }\end{array}$ & 498 \\
\hline
\end{tabular}




\begin{tabular}{|c|c|c|c|c|c|}
\hline 42 . & \begin{tabular}{|l} 
Synergism between \\
natural products and \\
antibiotics against \\
infectious diseases
\end{tabular} & Hemaiswarya S., Kruthiventi A.K., Doble M. & 2008 & Phytomedicine & 489 \\
\hline 43. & $\begin{array}{l}\text { Lead toxicity, a review of } \\
\text { the literature. Part I: } \\
\text { Exposure, evaluation, } \\
\text { and treatment }\end{array}$ & Patrick L. & 2006 & $\begin{array}{l}\text { Alternative } \\
\text { Medicine } \\
\text { Review }\end{array}$ & 481 \\
\hline 44. & $\begin{array}{l}\text { Complementary and } \\
\text { alternative medicine use } \\
\text { in Australia: A national } \\
\text { population-based survey }\end{array}$ & Xue C.C.L., Zhang A.L., Lin V., Da Costa C., Story D.F. & 2007 & $\begin{array}{l}\text { Journal of } \\
\text { Alternative and } \\
\text { Complementary } \\
\text { Medicine }\end{array}$ & 460 \\
\hline 45. & $\begin{array}{l}\text { Traditional Chinese } \\
\text { medicine network } \\
\text { pharmacology: Theory, } \\
\text { methodology and } \\
\text { application }\end{array}$ & Li S., Zhang B. & 2013 & $\begin{array}{l}\text { Chinese Journal } \\
\text { of Natural } \\
\text { Medicines }\end{array}$ & 455 \\
\hline 46. & $\begin{array}{l}\text { Beneficial effects of } \\
\text { green tea: A literature } \\
\text { review }\end{array}$ & Chacko S.M., Thambi P.T., Kuttan R., Nishigaki I. & 2010 & $\begin{array}{l}\text { Chinese } \\
\text { Medicine }\end{array}$ & 454 \\
\hline 47. & $\begin{array}{l}\text { Therapeutic applications } \\
\text { of pomegranate (Punica } \\
\text { granatum L.): A review }\end{array}$ & Jurenka J. & 2008 & $\begin{array}{l}\text { Alternative } \\
\text { Medicine } \\
\text { Review }\end{array}$ & 451 \\
\hline 48. & $\begin{array}{l}\text { Recent extraction } \\
\text { techniques for natural } \\
\text { products: Microwave- } \\
\text { assisted extraction and } \\
\text { pressurised solvent } \\
\text { extraction }\end{array}$ & Kaufmann B., Christen P. & 2002 & $\begin{array}{l}\text { Phytochemical } \\
\text { Analysis }\end{array}$ & 447 \\
\hline 49. & $\begin{array}{l}\text { Astaxanthin, a carotenoid } \\
\text { with potential in human } \\
\text { health and nutrition }\end{array}$ & Hussein G., Sankawa U., Goto H., Matsumoto K., Watanabe H. & 2006 & $\begin{array}{l}\text { Journal of } \\
\text { Natural } \\
\text { Products }\end{array}$ & 429 \\
\hline 50 . & $\begin{array}{l}\text { Antimicrobial activity of } \\
\text { essential oils: A 1976- } \\
1986 \text { literature review. } \\
\text { Aspects of the test } \\
\text { methods }\end{array}$ & Janssen A.M., Scheffer J.J.C., Baerheim Svendsen A. & 1987 & Planta Medica & 429 \\
\hline 51. & $\begin{array}{l}\text { Antioxidant activity, total } \\
\text { phenolic and total } \\
\text { flavonoid contents of } \\
\text { whole plant extracts } \\
\text { Torilis leptophylla L }\end{array}$ & Saeed N., Khan M.R., Shabbir M. & 2012 & $\begin{array}{l}\text { BMC } \\
\text { Complementary } \\
\text { and Alternative } \\
\text { Medicine }\end{array}$ & 426 \\
\hline 52. & $\begin{array}{l}\text { Clinical applications of } \\
\text { N-acetylcysteine }\end{array}$ & Kelly G.S. & 1998 & $\begin{array}{l}\text { Alternative } \\
\text { Medicine } \\
\text { Review }\end{array}$ & 422 \\
\hline
\end{tabular}




\begin{tabular}{|c|c|c|c|c|c|}
\hline 53. & $\begin{array}{l}\text { Nutritional quality of } \\
\text { organic versus } \\
\text { conventional fruits, } \\
\text { vegetables, and grains }\end{array}$ & Worthington $\mathrm{V}$. & 2001 & $\begin{array}{l}\text { Journal of } \\
\text { Alternative and } \\
\text { Complementary } \\
\text { Medicine }\end{array}$ & 421 \\
\hline 54. & $\begin{array}{l}\text { Chemotherapy-associated } \\
\text { oxidative stress: Impact } \\
\text { on chemotherapeutic } \\
\text { effectiveness }\end{array}$ & Conklin K.A. & 2004 & $\begin{array}{l}\text { Integrative } \\
\text { Cancer } \\
\text { Therapies }\end{array}$ & 419 \\
\hline 55. & Iridoids. a review & El-Naggar L.J., Beal J.L. & 1980 & $\begin{array}{l}\text { Journal of } \\
\text { Natural } \\
\text { Products }\end{array}$ & 418 \\
\hline 56. & $\begin{array}{l}\text { Anti-aids agents, } 11 . \\
\text { betulinic acid and } \\
\text { platanic acid as anti-HIV } \\
\text { principles from syzigium } \\
\text { claviflorum, and the anti- } \\
\text { HIV activity of } \\
\text { structurally related } \\
\text { triterpenoids }\end{array}$ & $\begin{array}{l}\text { Fujioka T., Kashiwada Y., Kilkuskie R.E., Cosentino L.M., } \\
\text { Bailas L.M., Jiang J.B., Janzen W.P., Chen I.-S., Lee K.-H. }\end{array}$ & 1994 & \begin{tabular}{|l} 
Journal of \\
Natural \\
Products
\end{tabular} & 415 \\
\hline 57. & $\begin{array}{l}\text { Bleomycin: New } \\
\text { perspectives on the } \\
\text { mechanism of action }\end{array}$ & Hecht S.M. & 2000 & $\begin{array}{l}\text { Journal of } \\
\text { Natural } \\
\text { Products }\end{array}$ & 406 \\
\hline 58. & $\begin{array}{l}\text { Plant-derived leading } \\
\text { compounds for } \\
\text { chemotherapy of human } \\
\text { immunodeficiency virus } \\
\text { (HIV) infection }\end{array}$ & Vlietinck A.J., De Bruyne T., Apers S., Pieters L.A. & 1998 & Planta Medica & 404 \\
\hline 59. & $\begin{array}{l}\text { Antioxidants and cancer } \\
\text { III: Quercetin }\end{array}$ & Lamson D.W., Brignall M.S. & 2000 & $\begin{array}{l}\text { Alternative } \\
\text { Medicine } \\
\text { Review }\end{array}$ & 403 \\
\hline 60. & $\begin{array}{l}\text { A microwell cytotoxicity } \\
\text { assay using Artemia } \\
\text { salina (brine shrimp) }\end{array}$ & $\begin{array}{l}\text { Solis P.N., Wright C.W., Anderson M.M., Gupta M.P., } \\
\text { Phillipson J.D. }\end{array}$ & 1993 & Planta Medica & 403 \\
\hline 61. & $\begin{array}{l}\text { How many cancer } \\
\text { patients use } \\
\text { complementary and } \\
\text { alternative medicine: A } \\
\text { systematic review and } \\
\text { metaanalysis }\end{array}$ & $\begin{array}{l}\text { Horneber M., Bueschel G., Dennert G., Less D., Ritter E., } \\
\text { Zwahlen M. }\end{array}$ & 2012 & $\begin{array}{l}\text { Integrative } \\
\text { Cancer } \\
\text { Therapies }\end{array}$ & 395 \\
\hline 62. & $\begin{array}{l}\text { The BBC survey of } \\
\text { complementary medicine } \\
\text { use in the UK }\end{array}$ & Ernst E., White A. & 2000 & $\begin{array}{l}\text { Complementary } \\
\text { Therapies in } \\
\text { Medicine }\end{array}$ & 395 \\
\hline 63. & $\begin{array}{l}\text { The scientific rediscovery } \\
\text { of an ancient Chinese }\end{array}$ & Zhu J.-S., Halpern G.M., Jones K. & 1998 & $\begin{array}{l}\text { Journal of } \\
\text { Alternative and }\end{array}$ & 389 \\
\hline
\end{tabular}




\begin{tabular}{|c|c|c|c|c|c|}
\hline & $\begin{array}{l}\text { herbal medicine: } \\
\text { Cordyceps sinensis part I }\end{array}$ & & & $\begin{array}{l}\text { Complementary } \\
\text { Medicine }\end{array}$ & \\
\hline 64. & $\begin{array}{l}\text { Therapeutic applications } \\
\text { of whey protein }\end{array}$ & Marshall K. & 2004 & $\begin{array}{l}\text { Alternative } \\
\text { Medicine } \\
\text { Review }\end{array}$ & 383 \\
\hline 65. & $\begin{array}{l}\text { Chemical toxins: A } \\
\text { hypothesis to explain the } \\
\text { global obesity epidemic }\end{array}$ & Baillie-Hamilton P.F. & 2002 & $\begin{array}{l}\text { Journal of } \\
\text { Alternative and } \\
\text { Complementary } \\
\text { Medicine }\end{array}$ & 377 \\
\hline 66. & $\begin{array}{l}\text { Quantitative 1H NMR: } \\
\text { Development and } \\
\text { potential of a method for } \\
\text { natural products analysis }\end{array}$ & Pauli G.F., Jaki B.U., Lankin D.C. & 2005 & $\begin{array}{l}\text { Journal of } \\
\text { Natural } \\
\text { Products }\end{array}$ & 376 \\
\hline 67. & $\begin{array}{l}\text { Antimicrobial activity of } \\
\text { some ethnomedicinal } \\
\text { plants used by Paliyar } \\
\text { tribe from Tamil Nadu, } \\
\text { India }\end{array}$ & Duraipandiyan V., Ayyanar M., Ignacimuthu S. & 2006 & $\begin{array}{l}\text { BMC } \\
\text { Complementary } \\
\text { and Alternative } \\
\text { Medicine }\end{array}$ & 375 \\
\hline 68. & $\begin{array}{l}\text { Goji (Lycium barbarum } \\
\text { and L. chinense): } \\
\text { Phytochemistry, } \\
\text { pharmacology and safety } \\
\text { in the perspective of } \\
\text { traditional uses and } \\
\text { recent popularity }\end{array}$ & Potterat O. & 2010 & Planta Medica & 374 \\
\hline 69. & $\begin{array}{l}\text { Constituents of cannabis } \\
\text { sativa l. xvii. a review of } \\
\text { the natural constituents }\end{array}$ & Turner C.E., Elsohly M.A., Boeren E.G. & 1980 & $\begin{array}{l}\text { Journal of } \\
\text { Natural } \\
\text { Products }\end{array}$ & 373 \\
\hline 70. & $\begin{array}{l}\text { Recent natural products } \\
\text { based drug development: } \\
\text { A pharmaceutical } \\
\text { industry perspective }\end{array}$ & Shu Y.-Z. & 1998 & $\begin{array}{l}\text { Journal of } \\
\text { Natural } \\
\text { Products }\end{array}$ & 371 \\
\hline 71. & $\begin{array}{l}\text { Cyclooxygenase } \\
\text { inhibitory and } \\
\text { antioxidant cyanidin } \\
\text { glycosides in cherries } \\
\text { and berries }\end{array}$ & Seeram N.P., Momin R.A., Nair M.G., Bourquin L.D. & 2001 & Phytomedicine & 370 \\
\hline 72. & $\begin{array}{l}\text { Zingiberis rhizoma: A } \\
\text { comprehensive review on } \\
\text { the ginger effect and } \\
\text { efficacy profiles }\end{array}$ & Chrubasik S., Pittler M.H., Roufogalis B.D. & 2005 & Phytomedicine & 368 \\
\hline 73. & $\begin{array}{l}\text { Ayurveda and traditional } \\
\text { Chinese medicine: A } \\
\text { comparative overview }\end{array}$ & Patwardhan B., Warude D., Pushpangadan P., Bhatt N. & 2005 & $\begin{array}{l}\text { Evidence-based } \\
\text { Complementary } \\
\text { and Alternative } \\
\text { Medicine }\end{array}$ & 366 \\
\hline
\end{tabular}




\begin{tabular}{|c|c|c|c|c|c|}
\hline 74. & $\begin{array}{l}\text { A-type proanthocyanidin } \\
\text { trimers from cranberry } \\
\text { that inhibit adherence of } \\
\text { uropathogenic P- } \\
\text { fimbriated Escherichia } \\
\text { coli }\end{array}$ & Foo L.Y., Lu Y., Howell A.B., Vorsa N. & 2000 & \begin{tabular}{|l} 
Journal of \\
Natural \\
Products
\end{tabular} & 366 \\
\hline 75. & $\begin{array}{l}\text { Historical review of } \\
\text { medicinal plants' usage }\end{array}$ & Petrovska B.B. & 2012 & $\begin{array}{l}\text { Pharmacognosy } \\
\text { Reviews }\end{array}$ & 363 \\
\hline 76. & $\begin{array}{l}\text { Plant sources of } \\
\text { hepatotoxic pyrrolizidine } \\
\text { alkaloids }\end{array}$ & Smith L.W., Culvenor C.C.J. & 1981 & $\begin{array}{l}\text { Journal of } \\
\text { Natural } \\
\text { Products }\end{array}$ & 361 \\
\hline 77. & $\begin{array}{l}\text { Steaming of ginseng at } \\
\text { high temperature } \\
\text { enhances biological } \\
\text { activity }\end{array}$ & $\begin{array}{l}\text { Wang Yu Kim, Jong Moon Kim, Sang Beom Han, Seung Ki Lee, } \\
\text { Nak Doo Kim, Park M.K., Chong Kook Kim, Park J.H. }\end{array}$ & 2000 & $\begin{array}{l}\text { Journal of } \\
\text { Natural } \\
\text { Products }\end{array}$ & 360 \\
\hline 78. & $\begin{array}{l}\text { Recent trends and } \\
\text { important developments } \\
\text { in propolis research }\end{array}$ & Bankova V. & 2005 & $\begin{array}{l}\text { Evidence-based } \\
\text { Complementary } \\
\text { and Alternative } \\
\text { Medicine }\end{array}$ & 357 \\
\hline 79. & $\begin{array}{l}\text { Therapeutic applications } \\
\text { of fenugreek }\end{array}$ & Basch E., Ulbricht C., Kuo G., Szapary P., Smith M. & 2003 & $\begin{array}{l}\text { Alternative } \\
\text { Medicine } \\
\text { Review }\end{array}$ & 357 \\
\hline 80. & $\begin{array}{l}\text { Antioxidant and free } \\
\text { radical scavenging } \\
\text { activity of Spondias } \\
\text { pinnata }\end{array}$ & Hazra B., Biswas S., Mandal N. & 2008 & $\begin{array}{l}\text { BMC } \\
\text { Complementary } \\
\text { and Alternative } \\
\text { Medicine }\end{array}$ & 354 \\
\hline 81. & $\begin{array}{l}\text { Antioxidant and radical } \\
\text { scavenging effects of } \\
\text { aged garlic extract and } \\
\text { its constituents }\end{array}$ & $\begin{array}{l}\text { Imai J., Ide N., Nagae S., Moriguchi T., Matsuura H., Itakura } \\
\text { Y. }\end{array}$ & 1994 & Planta Medica & 350 \\
\hline 82. & $\begin{array}{l}\text { Pentacyclic triterpenes of } \\
\text { the lupane, oleanane and } \\
\text { ursane group as tools in } \\
\text { cancer therapy }\end{array}$ & Laszczyk M.N. & 2009 & Planta Medica & 346 \\
\hline 83. & $\begin{array}{l}\text { Antioxidant and } \\
\text { antimicrobial activity of } \\
\text { Foeniculum vulgare and } \\
\text { crithmum maritimum } \\
\text { essential oils }\end{array}$ & Ruberto G., Baratta M.T., Deans S.G., Dorman H.J.D. & 2000 & Planta Medica & 346 \\
\hline 84. & $\begin{array}{l}\text { Medicinal plants used by } \\
\text { traditional healers in } \\
\text { Kancheepuram District of } \\
\text { Tamil Nadu, India }\end{array}$ & Muthu C., Ayyanar M., Raja N., Ignacimuthu S. & 2006 & $\begin{array}{l}\text { Journal of } \\
\text { Ethnobiology } \\
\text { and } \\
\text { Ethnomedicine }\end{array}$ & 344 \\
\hline & Lead toxicity part II: The & Patrick L. & 2006 & Alternative & 343 \\
\hline
\end{tabular}




\begin{tabular}{|c|c|c|c|c|c|}
\hline 85. & $\begin{array}{l}\text { role of free radical } \\
\text { damage and the use of } \\
\text { antioxidants in the } \\
\text { pathology and treatment } \\
\text { of lead toxicity }\end{array}$ & & & $\begin{array}{l}\text { Medicine } \\
\text { Review }\end{array}$ & \\
\hline 86. & $\begin{array}{l}\text { A rapid and effective } \\
\text { method for RNA } \\
\text { extraction from different } \\
\text { tissues of grapevine and } \\
\text { other woody plants }\end{array}$ & Gambino G., Perrone I., Gribaudo I. & 2008 & $\begin{array}{l}\text { Phytochemical } \\
\text { Analysis }\end{array}$ & 341 \\
\hline 87. & $\begin{array}{l}\text { Benefits and } \\
\text { requirements of vitamin } \\
\text { D for optimal health: A } \\
\text { review }\end{array}$ & Grant W.B., Holick M.F. & 2005 & $\begin{array}{l}\text { Alternative } \\
\text { Medicine } \\
\text { Review }\end{array}$ & 341 \\
\hline 88. & $\begin{array}{l}\text { Antimicrobial and } \\
\text { antioxidant activities of } \\
\text { three Mentha species } \\
\text { essential oils }\end{array}$ & $\begin{array}{l}\text { Mimica-Dukić N., Božin B., Soković M., Mihajlović B., } \\
\text { Matavulj M. }\end{array}$ & 2003 & Planta Medica & 338 \\
\hline 89. & The taxane diterpenoids & Baloglu E., Kingston D.G.I. & 1999 & $\begin{array}{l}\text { Journal of } \\
\text { Natural } \\
\text { Products }\end{array}$ & 337 \\
\hline 90. & $\begin{array}{l}\text { Flavonoids: A versatile } \\
\text { source of anticancer } \\
\text { drugs }\end{array}$ & Chahar M.K., Sharma N., Dobhal M.P., Joshi Y.C. & 2011 & $\begin{array}{l}\text { Pharmacognosy } \\
\text { Reviews }\end{array}$ & 332 \\
\hline 91. & $\begin{array}{l}\text { Resilience: A historical } \\
\text { review of the construct }\end{array}$ & Tusaie K., Dyer J. & 2004 & $\begin{array}{l}\text { Holistic } \\
\text { Nursing } \\
\text { Practice }\end{array}$ & 332 \\
\hline 92. & $\begin{array}{l}\alpha \text {-glucosidase inhibitors } \\
\text { from plants: A natural } \\
\text { approach to treat } \\
\text { diabetes }\end{array}$ & Kumar S., Narwal S., Kumar V., Prakash O. & 2011 & $\begin{array}{l}\text { Pharmacognosy } \\
\text { Reviews }\end{array}$ & 331 \\
\hline 93. & $\begin{array}{l}\text { Flavonoids from } \\
\text { Hypericum perforatum } \\
\text { show antidepressant } \\
\text { activity in the forced } \\
\text { swimming test }\end{array}$ & Butterweck V., Jürgenliemk G., Nahrstedt A., Winterhoff H. & 2000 & Planta Medica & 328 \\
\hline 94. & $\begin{array}{l}\text { Stigmasterols from typha } \\
\text { latifolia }\end{array}$ & Greca M.D., Monaco P., Previtera L. & 1990 & $\begin{array}{l}\text { Journal of } \\
\text { Natural } \\
\text { Products }\end{array}$ & 327 \\
\hline 95. & $\begin{array}{l}\text { Flavonoids and phenolic } \\
\text { acids: Role and } \\
\text { biochemical activity in } \\
\text { plants and human }\end{array}$ & Ghasemzadeh A., Ghasemzadeh N. & 2011 & $\begin{array}{l}\text { Journal of } \\
\text { Medicinal Plant } \\
\text { Research }\end{array}$ & 326 \\
\hline 96. & $\begin{array}{l}\text { Alternative antimicrobial } \\
\text { approach: Nano- }\end{array}$ & Beyth N., Houri-Haddad Y., Domb A., Khan W., Hazan R. & 2015 & $\begin{array}{l}\text { Evidence-based } \\
\text { Complementary }\end{array}$ & 324 \\
\hline
\end{tabular}




\begin{tabular}{|c|c|c|c|c|c|}
\hline & antimicrobial materials & & & $\begin{array}{l}\text { and Alternative } \\
\text { Medicine }\end{array}$ & \\
\hline 97. & $\begin{array}{l}\text { Anti-inflammatory } \\
\text { activity of linalool and } \\
\text { linalyl acetate } \\
\text { constituents of essential } \\
\text { oils }\end{array}$ & $\begin{array}{l}\text { Peana A.T., D'Aquila P.S., Panin F., Serra G., Pippia P., } \\
\text { Moretti M.D.L. }\end{array}$ & 2002 & Phytomedicine & 324 \\
\hline 98. & $\begin{array}{l}\text { Immunostimulant agents } \\
\text { from andrographis } \\
\text { paniculata }\end{array}$ & $\begin{array}{l}\text { Puri A., Saxena R., Saxena R.P., Saxena K.C., Srivastava V., } \\
\text { Tandon J.S. }\end{array}$ & 1993 & $\begin{array}{l}\text { Journal of } \\
\text { Natural } \\
\text { Products }\end{array}$ & 324 \\
\hline 99. & $\begin{array}{l}\text { The health benefits of } \\
\text { yoga and exercise: A } \\
\text { review of comparison } \\
\text { studies }\end{array}$ & Ross A., Thomas S. & 2010 & $\begin{array}{l}\text { Journal of } \\
\text { Alternative and } \\
\text { Complementary } \\
\text { Medicine }\end{array}$ & 320 \\
\hline 100. & $\begin{array}{l}\text { Anti-inflammatory } \\
\text { compounds of plant } \\
\text { origin. Part II. } \\
\text { Modulation of pro- } \\
\text { inflammatory cytokines, } \\
\text { chemokines and adhesion } \\
\text { molecules }\end{array}$ & Calixto J.B., Campos M.M., Otuki M.F., Santos A.R.S. & 2004 & Planta Medica & 319 \\
\hline
\end{tabular}

\section{Figures}

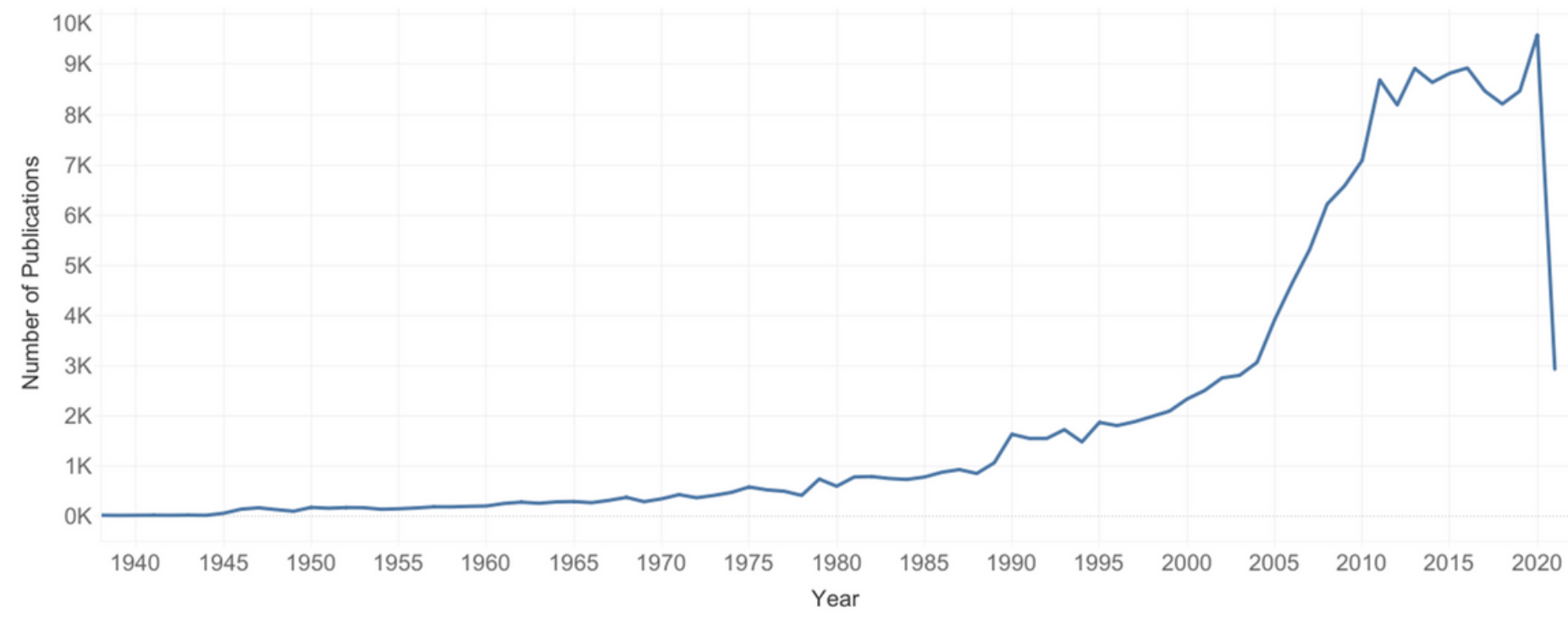

Figure 1

Number of Publications in Scopus-Indexed CAIM Journals per Year from 1938-2021 


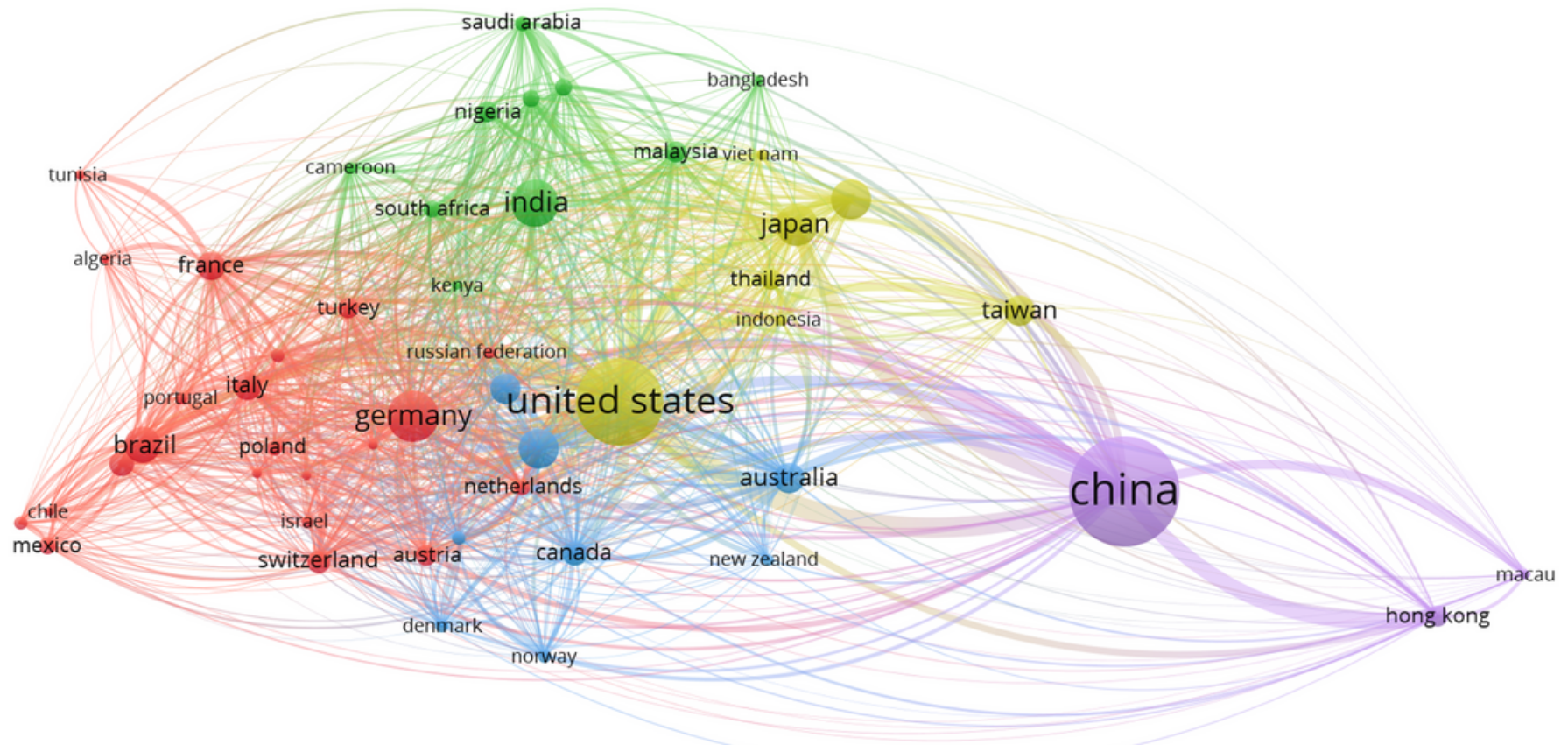

\& VOSviewer

Figure 2

Co-Authorship Analysis of the 50 Most Productive Countries

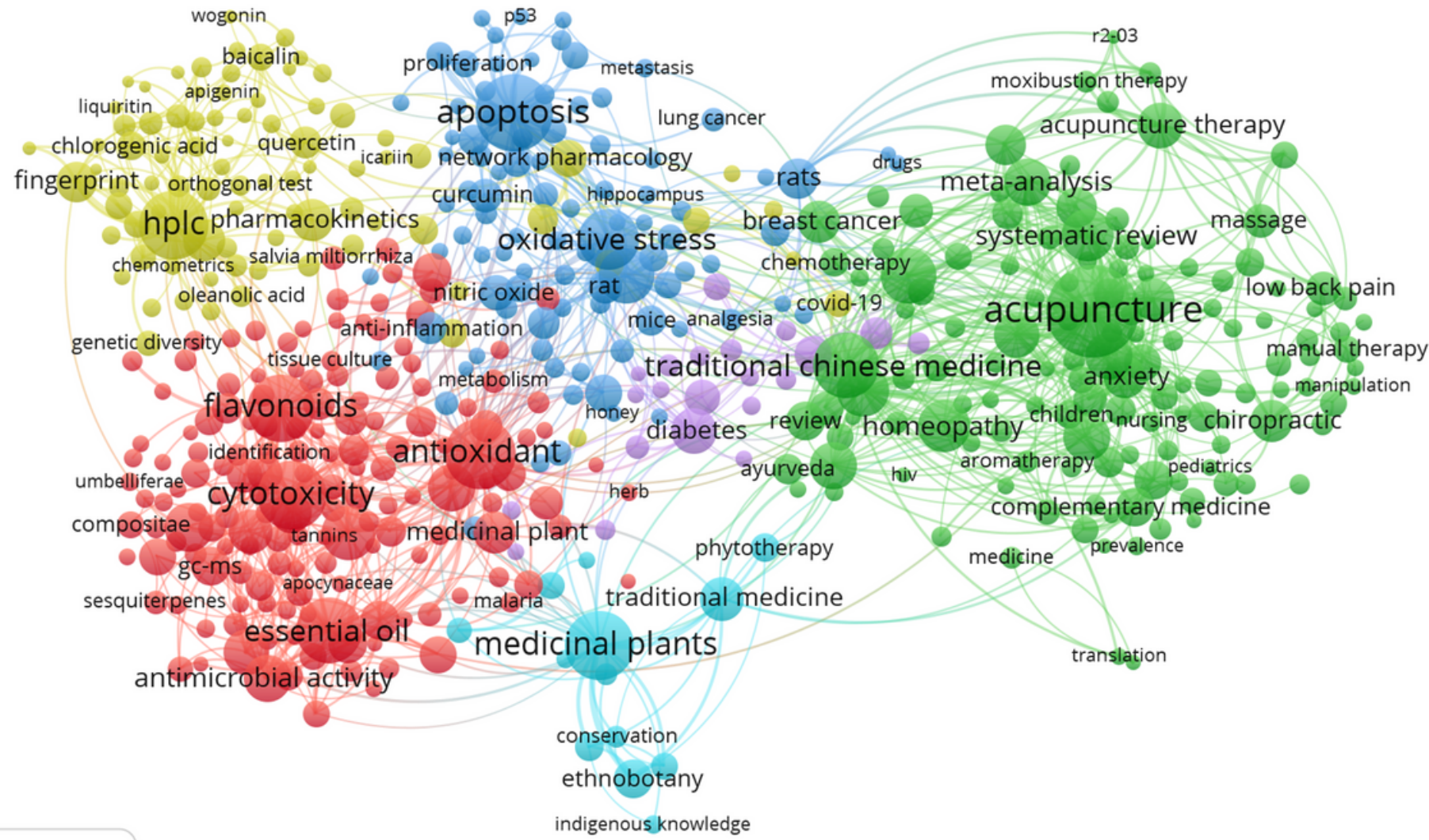

\& \& Vosviewer

Figure 3

Co-Occurrence Analysis of the 500 Most Frequent Author Keywords 


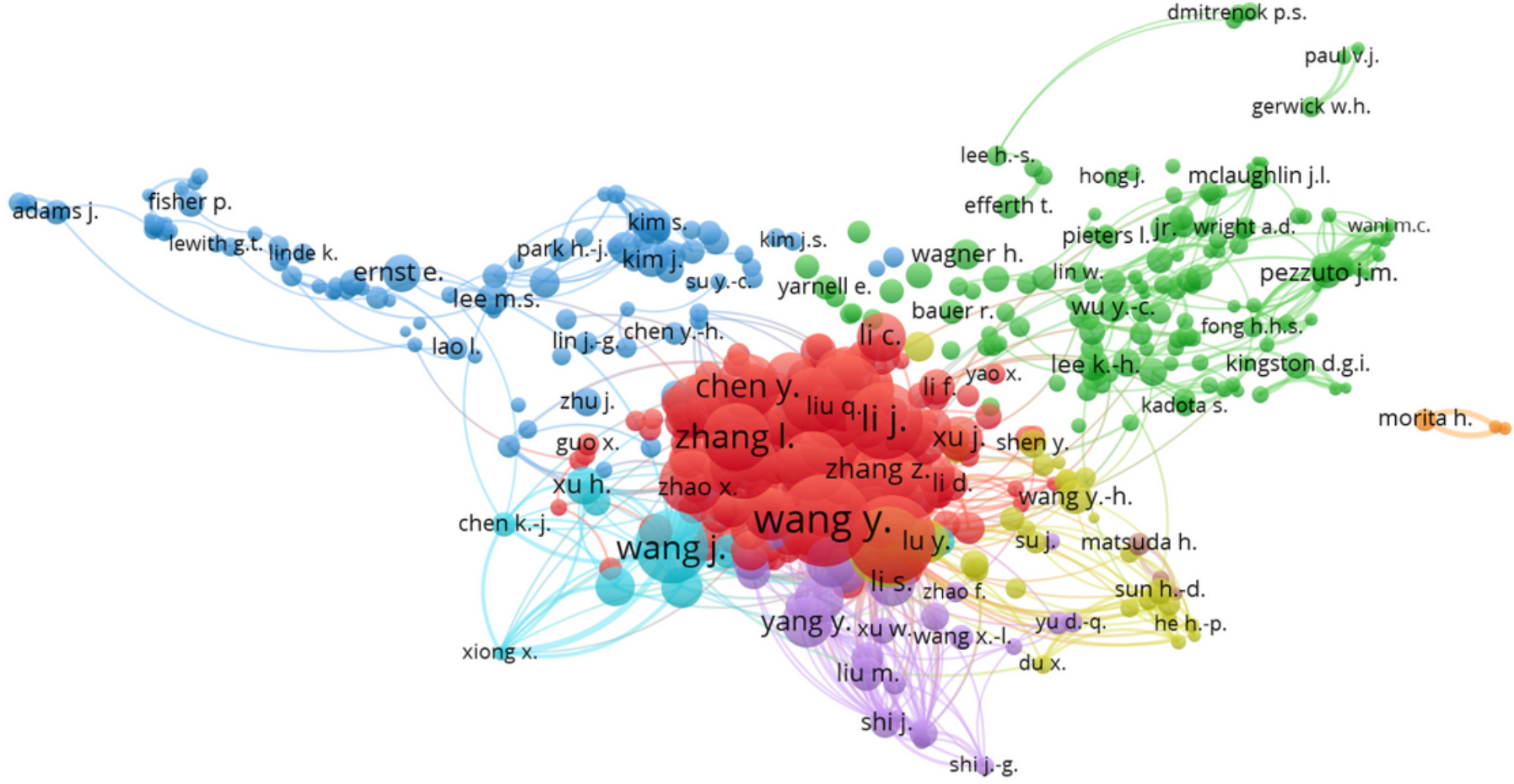

\section{\& VOSviewer}

\section{Figure 4}

Citation Analysis of the 500 Highest-Cited Authors

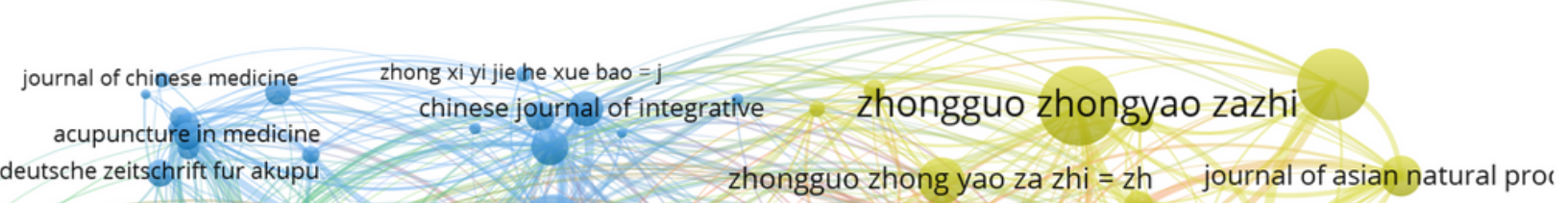

Jurnal of the american osteop

\section{evidence-based complementary a}
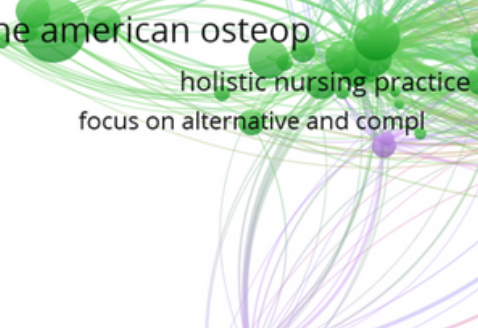

british homoeopathic journal

\& VOSviewer

\section{Figure 5}

Citation Analysis of the 100 CAIM Journals Publishing the Largest Number of Publications 